\title{
Analytical Method and Semianalytical Method for Analysis of Scattering by Anisotropic Sphere: A Review
}

\author{
Chao Wan ${ }^{1}$ and Hongning $\mathrm{Li}^{2}$ \\ ${ }^{1}$ Department of Electrical and Computer Engineering, National University of Singapore, Kent Ridge, Singapore 117583 \\ ${ }^{2}$ State Key Laboratory of Integrated Service Networks, Xidian University, Xi'an, Shaanxi 710071, China \\ Correspondence should be addressed to Chao Wan, wanchao@nus.edu.sg
}

Received 14 July 2011; Accepted 3 November 2011

Academic Editor: Joshua Le-Wei Li

Copyright (C 2012 C. Wan and H. Li. This is an open access article distributed under the Creative Commons Attribution License, which permits unrestricted use, distribution, and reproduction in any medium, provided the original work is properly cited.

\begin{abstract}
The history of methods for the electromagnetic scattering by an anisotropic sphere has been reviewed. Two main methods, angular expansion method and T-matrix method, which are widely used for the anisotropic sphere, are expressed in Cartesian coordinate firstly. The comparison of those and the further exploration on the scattering field are illustrated afterwards. Based on the most general form concluded by variable separation method, the coupled electric field and magnetic field of radial anisotropic sphere can be derived. By simplifying the condition, simpler case of uniaxial anisotropic media is expressed with confirmed coefficients for the internal and external field. Details of significant phenomenon are presented.
\end{abstract}

\section{Introduction}

Since the properties of isotropic homogeneous sphere is formulated by Lorentz [1] and Mie [2], due to plenty of corresponding significant phenomena [3-6] and increasing applications in atmospheric optics, remote sensing, computational electromagnetic and photonics, electromagnetic wave propagation and scattering for a sphere has been a subject of great interest in recent years. The amazing properties of solid anisotropic materials in nature and classes of artificial metamaterials of anisotropy would possibly produce plentiful technological applications. In the meantime, fabrication technology development has greatly enlarged the area of the anisotropic materials.

Cartesian anisotropic medium and Radial anisotropic medium are considered as two classic kinds of anisotropic materials [7]. Some novel properties of Cartesian anisotropy medium have been studied: magnetic plasma realizing the subdiffraction imaging [8], optical cavity made by indefinite medium of anisotropy [9], the sharply asymmetric reflection (SAR) effect [10], formation of tunable resonant photonic band gaps [11], negative-refractive behavior manipulation [12], and self-guiding unidirectional electromagnetics edge [13]. Based on the general anisotropic materials, those amazing properties can greatly enlarge the possibility to control light and wave in special structure and also open a new research area in the interaction between the electromagnetic wave and materials. For radial anisotropic materials, the spherical particle is of great interest [14-16]. The radial anisotropic particle scattering provides an insight into the interaction between the microparticles and the microwave or optical illumination by external devices [17-19], which could help to detect the position for some abnormal proteins [7, 20]. The classic spherical cloaks have been achieved by radial anisotropic materials [21]. The surface plasma resonance invisibility and extraordinary scattering of the RA particle provide a good opportunity to study the physical phenomena of microparticles.

For most of these published works, many properties of anisotropic material have been explored and applied. However, little work has been done so far on summaries and analysis for the methods to deal with problems of such anisotropic material. Scientists and engineers have to check the anisotropic class first and then choose a convenient numerical method or some commercial software. Either way will encounter redundant difficulties: for numerical method like FDTD, FEM, MOM, and other improved ones [22], prediction of the properties of medium is quite a difficulty, let alone the new structure design; for commercial software, the methods embedded in them are commonly standard, which are not able to deal with the possible novel materials 
with abnormal parameters. Therefore, modeling of those novel materials becomes extremely difficult. Fortunately, the analytical method and semianalytical method such as angular expansion method and T-matrix method will be very helpful in concluding good predictions for the anisotropic problems while keeping the accuracy [23-33].

It is of major purpose to summarize the analytical and semianalytical methods and their material parameter's effects on the scattering patterns, which will be of great use in electromagnetic wave properties for Cartesian and the Radial anisotropic spheres. In Section 2, besides two general analytical and semianalytical algorithms of anisotropy in Cartesian are reviewed, advantages and disadvantages of these two methods are also illustrated. In Section 3, the variables separation method for a single radial anisotropic sphere is demonstrated and numerical results obtained. A short conclusion is provided in Section 4.

\section{Electromagnetic Wave Scattering by a Cartesian Anisotropic Sphere}

2.1. Cartesian Anisotropy of Spheres. For Cartesian anisotropic medium, the constitutive relations are given by

$$
\bar{\varepsilon}=\varepsilon_{s}\left(\begin{array}{ccc}
\varepsilon_{r} & -i \varepsilon_{\kappa} & 0 \\
i \varepsilon_{\kappa} & \varepsilon_{r} & 0 \\
0 & 0 & 1
\end{array}\right) \quad \bar{\mu}=\mu_{s}\left(\begin{array}{ccc}
\mu_{r} & -i \mu_{\kappa} & 0 \\
i \mu_{\kappa} & \mu_{r} & 0 \\
0 & 0 & 1
\end{array}\right),
$$

where the identity dyadic is expressed as $\hat{\mathbf{I}}=\hat{\mathbf{x}} \widehat{\mathbf{x}}+\hat{\mathbf{y}} \widehat{\mathbf{y}}+\widehat{\mathbf{z}} \mathbf{\mathbf { z }}$. By extending Mie theory in isotropic cases, many methods such as FDTD, FEM, and MOM have been established to analyzed wave interaction issue in the medium. However, they cannot provide an explicit explanation for the physical phenomenon. Being able to deal with multilayered problems, the dyadic green's function is considered as one analytical approach [34]. However, the researchers have to encounter the singularities while they choose the integration path, then the expression turns to be rather complicated. In [35], Uzunoglu first employed the Fourier form to state the field, followed by a lot of work on this direction for its clear computation procedure [36, 37]. Using the angular expansion to present electromagnetic field, Ren successfully transferred the three-dimensional problem into a two-dimension integration [23]. The field can be treated as combination of infinite plane wave, while only four kinds of Bessel function need to be considered according to the wave direction. After that, Dr. Sarkar gives an explicit expression of the plane wave using vector spherical wave functions, where the field in the media is a form of that infinite plane wave $[38,39]$. Based on these clear field and plane wave expression, Dr. Geng proposed a transpicuous procedure and made a fundamental computation [24-28]. The same result has been achieved by other researcher when compared with Geng's work [29]. Besides, the method firstly dealing with the photonic band problem can be seen as another way [40]. Instead of giving an explicit expression and using the integral form on the surface, Lin used the transfer matrix method to gather the information in the medium [41]. By using the complete matrix form, they express the wave number $k$ by a matrix vector instead of an expression $[32,42]$, and this approach obtains great success [10-13]. Herein, we mainly focus on the review and analysis of these two analytical and semianalytical methods.

2.2. Angular Expansion Method. Inside the sourceless and homogeneous anisotropic sphere, the constitutive relationships as used in the Maxwell equations are

$$
\begin{gathered}
\nabla \times \mathbf{E}=i \omega \mathbf{B}, \\
\nabla \times \mathbf{H}=-i \omega \mathbf{D}, \\
\nabla \cdot \mathbf{D}=0, \\
\nabla \cdot \mathbf{B}=0 .
\end{gathered}
$$

The relations between the electric displacement vector $\mathbf{D}$, the magnetic induction $\mathbf{B}$, the electric field $\mathbf{E}$, and the magnetic field $\mathbf{H}$ inside the medium are given by

$$
\mathbf{D}=\bar{\varepsilon} \cdot \mathbf{E}, \quad \mathbf{B}=\bar{\mu} \cdot \mathbf{H}
$$

The parameters are defined in Cartesian coordinates as (1). We can rewrite the Maxwell's equations in a convenient form [27]:

$$
\nabla \times\left[\bar{\mu}^{-1} \cdot \nabla \times \mathbf{E}\right]-\omega^{2} \bar{\varepsilon} \cdot \mathbf{E}=0 .
$$

The solution to (7) can be written in the Fourier transform:

$$
\mathbf{E}(\mathbf{r})=\int_{-\infty}^{+\infty} d k_{x} \int_{-\infty}^{+\infty} d k_{y} \int_{-\infty}^{+\infty} \mathbf{E}(\mathbf{k}) e^{i \mathbf{k} \cdot \mathbf{r}} d k_{z}
$$

where the vector wave number is expressed as $\mathbf{k}=k_{x} \widehat{\mathbf{x}}+k_{y} \hat{\mathbf{y}}+$ $k_{z} \hat{\mathbf{z}}$, the space vector as $\mathbf{r}=x \hat{\mathbf{x}}+y \hat{\mathbf{y}}+z \hat{\mathbf{z}}$, and $\hat{\mathbf{x}}, \hat{\mathbf{y}}, \quad \hat{\mathbf{z}}$ being the unit vectors in Cartesian coordinates. Here, the problem is transferred from spatial domain to spectrum domain. For each point in the medium, the electric field $\mathbf{E}$ can be seen as a combination of infinite plane wave. Then, for any direction wave, we have $\nabla=i \mathbf{k}$. Substituting (8) into (7), the electric field equation is transformed into

$$
\int_{-\infty}^{+\infty} d k_{x} \int_{-\infty}^{+\infty} d k_{y} \int_{-\infty}^{+\infty} \overline{\mathbf{A}}(\mathbf{k}) \cdot \mathbf{E}(\mathbf{k}) e^{i \mathbf{k} \cdot \mathbf{r}} d k_{z}=0
$$

where

$$
\overline{\mathbf{A}}(\mathbf{k})=\left(\begin{array}{ccc}
-b_{1} k_{z}^{2}-b_{3} k_{z}^{2}+a_{1} & b_{3} k_{x} k_{y}-i b_{2} k_{z}^{2}-i a_{2} & b_{1} k_{x} k_{z}+i b_{2} k_{y} k_{z} \\
b_{3} k_{x} k_{y}-i b_{2} k_{z}^{2}-i a_{2} & -b_{1} k_{z}^{2}-b_{3} k_{z}^{2}+a_{1} & b_{1} k_{y} k_{z}-i b_{2} k_{x} k_{z} \\
b_{1} k_{x} k_{z}-i b_{2} k_{y} k_{z} & b_{1} k_{y} k_{z}+i b_{2} k_{x} k_{z} & -b_{1}\left(k_{x}^{2}+k_{y}^{2}\right)+a_{3}
\end{array}\right)
$$


with

$$
\begin{array}{cc}
a_{1}=\omega^{2} \varepsilon_{s} \varepsilon_{r} & a_{2}=\omega^{2} \varepsilon_{s} \varepsilon_{\kappa} \quad a_{3}=\omega^{2} \varepsilon_{s}, \\
b_{1}=\frac{\mu_{r}}{\mu_{s}\left(\mu_{r}^{2}-\mu_{\kappa}^{2}\right)} & b_{2}=\frac{\mu_{\kappa}}{\mu_{s}\left(\mu_{r}^{2}-\mu_{\kappa}^{2}\right)} \quad b_{3}=\frac{1}{\mu_{s}} .
\end{array}
$$

In order to make (9) exist all the time, we write the eigenvalue equation as

$$
\operatorname{Det}[\overline{\mathbf{A}}(\mathbf{k})]=0
$$

Here, the eigenvalue $k$ is a function of the angular $\left(\theta_{k}, \phi_{k}\right)$, the equation turns to be

$$
A\left(\theta_{k}, \phi_{k}\right) k^{4}-B\left(\theta_{k}, \phi_{k}\right) k^{2}+C=0,
$$

where

$$
\begin{aligned}
A\left(\theta_{k}, \phi_{k}\right)= & {\left[b_{1} b_{3} \sin ^{2} \theta_{k}+\left(b_{1}^{2}-b_{2}^{2}\right) \cos ^{2} \theta_{k}\right] } \\
& \times\left[a_{1} \sin ^{2} \theta_{k}+a_{3} \cos ^{2} \theta_{k}\right], \\
B\left(\theta_{k}, \phi_{k}\right)= & {\left[b_{1}\left(a_{1}^{2}-a_{2}^{2}\right)+b_{3} a_{1} a_{3}\right] \sin ^{2} \theta_{k} } \\
& +2 a_{3}\left(b_{1} a_{1}+b_{2} a_{2}\right) \cos ^{2} \theta_{k}, \\
C= & a_{3}\left(a_{1}^{2}-a_{2}^{2}\right), \\
k^{2}= & k_{x}^{2}+k_{y}^{2}+k_{z}^{2}, \\
\theta_{k}= & \tan ^{-1}\left(\frac{\sqrt{k_{x}^{2}+k_{y}^{2}}}{k_{z}}\right), \\
\phi_{k}= & \tan ^{-1}\left(\frac{k_{y}}{k_{x}}\right), \\
k_{1,3}^{2}= & \frac{B+\sqrt{B^{2}-4 A C}}{2 A}, \\
k_{2,4}^{2}= & \frac{B-\sqrt{B^{2}-4 A C}}{2 A},
\end{aligned}
$$

and the eigenvectors can also be obtained from (10) at the same time and are given as follows:

$$
\begin{aligned}
\mathbf{E}_{q} & =\mathbf{F}_{q}^{e} f_{q}\left(\theta_{k}, \phi_{k}\right) \\
& =\left[F_{q x}^{e}\left(\theta_{k}, \phi_{k}\right) \hat{\mathbf{x}}+F_{q y}^{e}\left(\theta_{k}, \phi_{k}\right) \hat{\mathbf{y}}+F_{q z}^{e}\left(\theta_{k}, \phi_{k}\right) \hat{\mathbf{z}}\right] f_{q}\left(\theta_{k}, \phi_{k}\right),
\end{aligned}
$$

where $q=1,2,3$, or 4 , here $\mathbf{E}_{q}$ is a plane wave, and

$$
\begin{gathered}
F_{q x}^{e}=-\frac{\Delta_{1}}{\Delta} \sin \phi_{k}+\frac{\Delta_{2}}{\Delta} \cos \phi_{k} \\
F_{q y}^{e}=\frac{\Delta_{1}}{\Delta} \cos \phi_{k}+\frac{\Delta_{2}}{\Delta} \sin \phi_{k}, \\
F_{q z}^{e}=1
\end{gathered}
$$

with

$$
\begin{aligned}
\Delta_{1}= & i\left(b_{1} a_{2}+b_{2} a_{1}\right) k_{q}^{2} \sin \theta_{k} \cos \theta_{k}, \\
\Delta_{2}= & {\left[b_{1} b_{3} k_{q}^{2} \sin ^{2} \theta_{k}+\left(b_{1}^{2}-b_{2}^{2}\right) k_{q}^{2} \cos ^{2} \theta_{k}\right] } \\
& \times k_{q}^{2} \sin \theta_{k} \cos \theta_{k}-\left(b_{1} a_{1}+b_{2} a_{2}\right) \\
\Delta= & -\left(b_{2} k_{q}^{2} \cos ^{2} \theta_{k}+a_{2}\right)^{2}+\left(b_{1} k_{q}^{2} \cos ^{2} \theta_{k}-a_{1}\right) \\
& \times\left(b_{1} k_{q}^{2} \cos ^{2} \theta_{k}+b_{3} k_{q}^{2} \sin ^{2} \theta_{k}-a_{1}\right) .
\end{aligned}
$$

Here, all the angular and eigenvalues should be considered. The E-field in (7) is then given as

$$
\mathbf{E}(r)=\sum_{q=1}^{2} \int_{0}^{\pi} \int_{0}^{2 \pi} F_{q}^{e}\left(\theta_{k}, \phi_{k}\right) f_{q}\left(\theta_{k}, \phi_{k}\right) e^{i \mathbf{k}_{q} \cdot \mathbf{r}} k_{q}^{2} \sin \theta_{k} d \theta_{k} d \phi_{k},
$$

$$
\mathbf{k}_{q}=k_{q} \sin \theta_{k} \cos \phi_{k} \hat{\mathbf{x}}+k_{q} \sin \theta_{k} \sin \phi_{k} \hat{\mathbf{y}}+k_{q} \cos \theta_{k} \hat{\mathbf{z}} .
$$

Only two roots need to be considered, while the periodic function $f_{q}\left(\theta_{k}, \phi_{k}\right)$ denoting the angular spectrum amplitude can be expressed as

$$
f_{q}\left(\theta_{k}, \phi_{k}\right)=\sum_{m^{\prime}, n^{\prime}} G_{m^{\prime} n^{\prime} q} P_{n^{\prime}}^{m^{\prime}}\left(\cos \theta_{k}\right) e^{i m^{\prime} \phi_{k}},
$$

where $P_{n}^{m}(x)$ is the associated Legendre function, and $\sum_{m^{\prime}, n^{\prime}}$ means that $n^{\prime}$ is from 0 to $+\infty$ and $m^{\prime}$ is from $-n^{\prime}$ to $n^{\prime}$. Substituting (20) to (18), we obtain

$$
\begin{aligned}
\mathbf{E}(r)= & \sum_{q=1}^{2} \sum_{m^{\prime}, n^{\prime}} G_{m^{\prime} n^{\prime} q} \int_{0}^{\pi} \int_{0}^{2 \pi} F_{q}^{e}\left(\theta_{k}, \phi_{k}\right) P_{n^{\prime}}^{m^{\prime}} \\
& \times\left(\cos \theta_{k}\right) e^{i m^{\prime} \phi_{k}} e^{i \mathbf{k}_{q} \cdot \mathbf{r}} k_{q}^{2} \sin \theta_{k} d \theta_{k} d \phi_{k} .
\end{aligned}
$$

Using the identity to stand for the part $e^{i \mathbf{k}_{q} \cdot \mathbf{r}}$,

$$
\begin{aligned}
e^{i \mathbf{k} \cdot \mathbf{r}}= & \sum_{n=0}^{\infty} i^{n}(2 n+1) j_{n}(k r) \\
& \times\left[\sum_{m=0}^{n} \frac{(n-m) !}{(n+m) !} P_{n}^{m}\left(\cos \theta_{k}\right) P_{n}^{m}(\cos \theta) e^{i m\left(\phi-\phi_{k}\right)}\right. \\
& \left.\quad+\sum_{m=0}^{n} \frac{(n-m) !}{(n+m) !} P_{n}^{m}\left(\cos \theta_{k}\right) P_{n}^{m}(\cos \theta) e^{-i m\left(\phi-\phi_{k}\right)}\right] .
\end{aligned}
$$

Substituting (22) into (21), we achieve the solution of $\mathbf{E}(\mathbf{r})$ for a general homogeneous gyrotropic anisotropic media. Then, the vector spherical wave functions is employed to express the eigenvector, which is one key step. After this, the internal field can be in the form of VSWFs:

$$
\begin{gathered}
\mathbf{F}_{q}^{e}(\theta, \phi) e^{i \mathbf{k}_{q} \cdot \mathbf{r}} \\
=\sum_{m, n}\left[A_{m n q}^{e}\left(\theta_{k}\right) \mathbf{M}_{m n}^{(1)}\left(r, k_{q}\right)+B_{m n q}^{e}\left(\theta_{k}\right) \mathbf{N}_{m n}^{(1)}\left(r, k_{q}\right)\right. \\
\left.+C_{m n q}^{e}\left(\theta_{k}\right) \mathbf{L}_{m n}^{(1)}\left(r, k_{q}\right)\right] e^{-i m \phi_{k}} .
\end{gathered}
$$


The other internal field parameters, $A_{m n q}^{e}\left(\theta_{k}\right), B_{m n q}^{e}\left(\theta_{k}\right)$, and $C_{m n q}^{e}\left(\theta_{k}\right)$ can be obtained and details are from $[27,33]$.

Inserting (23) into (21), and integrating with respect to $\phi_{k}$, we have

$$
\begin{aligned}
\mathbf{E}(r)= & \sum_{q=1}^{2} \sum_{m, n} \sum_{n^{\prime}} 2 \pi G_{m n^{\prime} q} \\
\times & \int_{0}^{\pi}\left[A_{m n q}^{e}\left(\theta_{k}\right) \mathbf{M}_{m n}^{(1)}\left(r, k_{q}\right)+B_{m n q}^{e}\left(\theta_{k}\right) \mathbf{N}_{m n}^{(1)}\left(r, k_{q}\right)\right. \\
& \left.+C_{m n q}^{e}\left(\theta_{k}\right) \mathbf{L}_{m n}^{(1)}\left(r, k_{q}\right)\right] \\
& \times P_{n^{\prime}}^{m^{\prime}}\left(\cos \theta_{k}\right) e^{i m^{\prime} \phi_{k}} k_{q}^{2} \sin \theta_{k} d \theta_{k} d \phi_{k},
\end{aligned}
$$

which is an eigenfunction representation of the E-field in gyrotropic anisotropic media. We can get the $\mathbf{H}$-field in a similar form $[27,33]$.

From the result in (24), it is found that we can use the VSWFs to present the field, and this procedure is also suitable for the external area. Afterwards, we take the plane wave as an example. Assuming that an incident plane wave is $\mathbf{E}=$ $\widehat{\mathbf{x}} E_{0} e^{i k_{0} z}$, the incident electromagnetic fields can be expanded by an infinite series of spherical vector wave functions for an isotropic medium as follows:

$$
\begin{aligned}
\mathbf{E}^{\text {inc }}= & E_{0} \sum_{n, m}\left[\delta_{m, 1}+\delta_{m,-1}\right] \\
& \times\left[a_{m n}^{x} \mathbf{M}_{m n}^{(1)}\left(k_{0}, \mathbf{r}\right)+b_{m n}^{x} \mathbf{N}_{m n}^{(1)}\left(k_{0}, \mathbf{r}\right)\right], \\
\mathbf{H}^{\text {inc }}= & \frac{k_{0}}{i \omega \mu_{0}} E_{0} \sum_{n, m}\left[\delta_{m, 1}+\delta_{m,-1}\right] \\
& \times\left[a_{m n}^{x} \mathbf{M}_{m n}^{(1)}\left(k_{0}, \mathbf{r}\right)+b_{m n}^{x} \mathbf{N}_{m n}^{(1)}\left(k_{0}, \mathbf{r}\right)\right],
\end{aligned}
$$

where

$$
\begin{aligned}
& a_{m n}^{x}= \begin{cases}i^{n+1} \frac{2 n+1}{2 n(n+1)}, & m=1, \\
i^{n+1} \frac{2 n+1}{2}, & m=-1,\end{cases} \\
& b_{m n}^{x}= \begin{cases}i^{n+1} \frac{2 n+1}{2 n(n+1)}, & m=1, \\
-i^{n+1} \frac{2 n+1}{2}, & m=-1,\end{cases} \\
& \delta_{s, l}= \begin{cases}1, \quad s=l, \\
0, \quad s \neq l,\end{cases}
\end{aligned}
$$

the scattering fields are expanded as

$$
\begin{aligned}
\mathbf{E}^{s} & =\sum_{n, m}\left[A_{m n}^{s} \mathbf{M}_{m n}^{(3)}\left(k_{0}, \mathbf{r}\right)+B_{m n}^{x} \mathbf{N}_{m n}^{(3)}\left(k_{0}, \mathbf{r}\right)\right], \\
\mathbf{H}^{s} & =\frac{k_{0}}{i \omega \mu_{0}} \sum_{n, m}\left[A_{m n}^{s} \mathbf{M}_{m n}^{(3)}\left(k_{0}, \mathbf{r}\right)+B_{m n}^{x} \mathbf{N}_{m n}^{(3)}\left(k_{0}, \mathbf{r}\right)\right],
\end{aligned}
$$

where $A_{m n}^{s}$ and $B_{m n}^{s}$ are unknown coefficients and stand for the external field information, and $k_{0}=\omega \sqrt{\mu_{0} \varepsilon_{0}}, \mu_{0}$ and $\mathcal{E}_{0}$ denote the free space, wave number, permeability, and permittivity. Until here, this method is being discussed in an unbounded material. We should match the boundary condition on the surface when we limit it to an anisotropic sphere. The tangential components of the electromagnetic field continues at $r=a$ and we have

$$
\begin{gathered}
\sum_{q=1}^{2} \sum_{n^{\prime}}^{\infty} 2 \pi G_{m n^{\prime} q} \int_{0}^{\pi} Q_{m n q} P_{n^{\prime}}^{m^{\prime}}\left(\cos \theta_{k}\right) k_{q}^{2} \sin \theta_{k} d \theta_{k} \\
=E_{0}\left[\delta_{m, 1}+\delta_{m,-1}\right] a_{m n}^{x} \cdot \frac{i}{\left(k_{0} a\right)^{2}}, \\
\sum_{q=1}^{2} \sum_{n^{\prime}}^{\infty} 2 \pi G_{m n^{\prime} q} \int_{0}^{\pi} R_{m n q} P_{n^{\prime}}^{m^{\prime}}\left(\cos \theta_{k}\right) k_{q}^{2} \sin \theta_{k} d \theta_{k} \\
=E_{0}\left[\delta_{m, 1}+\delta_{m,-1}\right] b_{m n}^{x} \cdot \frac{i}{\left(k_{0} a\right)^{2}},
\end{gathered}
$$

where

$$
\begin{aligned}
& Q_{m n q}=\left\{A_{m n q}^{e} \frac{1}{k_{0} r} \frac{d}{d r}\left[r h_{n}^{(1)}\left(k_{0} r\right)\right] j_{n}\left(k_{q} r\right)\right. \\
&-\frac{i \omega \mu_{0}}{k_{0}}\left[B_{m n q}^{h} \frac{1}{k_{q} r} \frac{d}{d r}\left[r j_{n}\left(k_{q} r\right)\right]+C_{m n q}^{h} \frac{j_{n}\left(k_{q} r\right)}{r}\right] \\
&\left.\cdot h_{n}^{(1)}\left(k_{0} r\right)\right\}_{r=a}, \\
& R_{m n q}=\left\{\frac{i \omega \mu_{0}}{k_{0}} A_{m n q}^{h} \frac{1}{k_{0} r} \frac{d}{d r}\left[r h_{n}^{(1)}\left(k_{0} r\right)\right] j_{n}\left(k_{q} r\right)\right. \\
&-\left[B_{m n q}^{e} \frac{1}{k_{q} r} \frac{d}{d r}\left[r j_{n}\left(k_{q} r\right)\right]+C_{m n q}^{e} \frac{j_{n}\left(k_{q} r\right)}{r}\right] \\
&\left.\cdot h_{n}^{(1)}\left(k_{0} r\right)\right\}_{r=a} \cdot
\end{aligned}
$$

The scattering coefficients $A_{m n}^{s}$ and $B_{m n}^{s}$, are thus expressed as

$$
\begin{aligned}
A_{m n}^{s}=\frac{1}{h_{n}^{(1)}\left(k_{0} a\right)} & {\left[\sum_{n^{\prime}=0}^{\infty} \sum_{q=1}^{2} 2 \pi G_{m n q} \int_{0}^{\pi} A_{m n q}^{e} j_{n}\left(k_{q} a\right)\right.} \\
& \times P_{n^{\prime \prime}}^{m^{\prime}}\left(\cos \theta_{k}\right) k_{q}^{2} \sin \theta_{k} d \theta_{k} \\
& \left.-E_{0}\left[\delta_{m, 1}+\delta_{m, 1}\right] a_{m n}^{x} j_{n}\left(k_{0} a\right)\right],
\end{aligned}
$$




$$
\begin{array}{r}
B_{m n}^{s}=\frac{1}{h_{n}^{(1)}\left(k_{0} a\right)}\left[\frac{i \omega \mu_{0}}{k_{0}} \sum_{n^{\prime}=0}^{\infty} \sum_{q=1}^{2} 2 \pi G_{m n q} \int_{0}^{\pi} A_{m n q}^{h} j_{n}\left(k_{q} a\right)\right. \\
\times P_{n^{\prime}}^{m^{\prime}}\left(\cos \theta_{k}\right) k_{q}^{2} \sin \theta_{k} d \theta_{k} \\
\left.-E_{0}\left[\delta_{m, 1}+\delta_{m, 1}\right] b_{m n}^{x} j_{n}\left(k_{0} a\right)\right] .
\end{array}
$$

Then the internal and external field can be expressed in the form of the coefficients.

2.3. T-Matrix Method. Different from the angular expansion method, here we use magnetic induction $\mathbf{B}$ to rewrite the Maxwell equation [32]:

$$
\nabla \times\left[\varepsilon_{s} \bar{\varepsilon}^{-1} \cdot\left(\nabla \times \mu_{s} \bar{\mu}^{-1} \cdot \mathbf{B}\right)\right]-k_{s}^{2} \mathbf{B}=0 .
$$

The divergenceless property (31) suggests that $\mathbf{B}$ be expanded in terms of the vector spherical wave functions $\mathbf{M}_{m n}^{(1)}(k, \mathbf{r})$ and $\mathbf{N}_{m n}^{(1)}(k, \mathbf{r})[32,41,42]:$

$$
\mathbf{B}=\sum_{n, m} \bar{E}_{m n}\left[d_{m n} \mathbf{M}_{m n}^{(1)}(k, \mathbf{r})+c_{m n} \mathbf{N}_{m n}^{(1)}(k, \mathbf{r})\right]
$$

where $k$ is not undetermined. As mentioned above, there are three kinds of VSWF's $\mathbf{M}_{m n}^{(1)}(k, \mathbf{r}), \mathbf{N}_{m n}^{(1)}(k, \mathbf{r})$ and $\mathbf{L}_{m n}^{(1)}(k, \mathbf{r})$. For this reason, (31) does not involve $\mathbf{L}_{m n}^{(1)}(k, \mathbf{r})$, because its curl is 0 , for details of the parameters see $[32,41,42]$. Since the VSWFs is a complete frame and can stand for all vectors in the space, we can use them to present the elements in (31), written as

$$
\begin{aligned}
\varepsilon_{s} \bar{\varepsilon}^{-1} \cdot \mathbf{M}_{m n}= & \sum_{v=0}^{+\infty} \sum_{u=-v}^{v}\left[\widetilde{o}_{u v}^{m n} \mathbf{M}_{u v}+\widetilde{p}_{u v}^{m n} \mathbf{N}_{u v}+\tilde{q}_{u v}^{m n} \mathbf{L}_{u v}\right], \\
\mathcal{\varepsilon}_{s} \bar{\varepsilon}^{-1} \cdot \mathbf{N}_{m n}= & \sum_{v=0}^{+\infty} \sum_{u=-v}^{v}\left[\bar{o}_{u v}^{m n} \mathbf{M}_{u v}+\bar{p}_{u v}^{m n} \mathbf{N}_{u v}+\bar{q}_{u v}^{m n} \mathbf{L}_{u v}\right] \\
\mu_{s} \bar{\mu}^{-1} \cdot \mathbf{M}_{m n}= & \sum_{v=0}^{+\infty} \sum_{u=-v}^{v}\left[\widetilde{g}_{u v}^{m n} \mathbf{M}_{u v}+\widetilde{e}_{u v}^{m n} \mathbf{N}_{u v}+\tilde{f}_{u v}^{m n} \mathbf{L}_{u v}\right] \\
& +\infty \\
\mu_{s} \bar{\mu}^{-1} \cdot \mathbf{N}_{m n}= & \sum_{v=0} \sum_{u=-v}^{v}\left[\bar{g}_{u v}^{m n} \mathbf{M}_{u v}+\bar{e}_{u v}^{m n} \mathbf{N}_{u v}+\bar{f}_{u v}^{m n} \mathbf{L}_{u v}\right]
\end{aligned}
$$

with

$$
\begin{aligned}
& \widetilde{o}_{u v}^{m n}=\delta_{n v} \delta_{m u}+\frac{\left[\left(n^{2}+n-m^{2}\right) \bar{\varepsilon}_{r}^{\prime}+m \varepsilon_{\kappa}^{\prime}\right] \delta_{n v} \delta_{m u}}{n(n+1)} \\
& \tilde{p}_{u v}^{m n}=\frac{i(n+m)\left[m \bar{\varepsilon}_{r}^{\prime}-(n+1) \varepsilon_{\kappa}^{\prime}\right] \delta_{n-1, v} \delta_{m u}}{n(2 n+1)}+\frac{i(n-m+1)\left[m \bar{\varepsilon}_{r}^{\prime}+n \varepsilon_{\kappa}^{\prime}\right] \delta_{n+11, v} \delta_{m u}}{(n+1)(2 n+1)}, \\
& \tilde{q}_{u v}^{m n}=\frac{-i(n+m)\left[m \bar{\varepsilon}_{r}^{\prime}-(n+1) \varepsilon_{\kappa}^{\prime}\right] \delta_{n-1, v} \delta_{m u}}{(2 n+1)}+\frac{i(n-m+1)\left[m \bar{\varepsilon}_{r}^{\prime}+n \varepsilon_{\kappa}^{\prime}\right] \delta_{n+11, v} \delta_{m u}}{(2 n+1)}, \\
& \bar{o}_{u v}^{m n}=-\frac{i(n+m)(n+1)\left[m \bar{\varepsilon}_{r}^{\prime}+(n-1) \varepsilon_{\kappa}^{\prime}\right] \delta_{n-1, v} \delta_{m u}}{n(n-1)(2 n+1)}-\frac{i(n-m+1) n\left[m \bar{\varepsilon}_{r}^{\prime}-(n+2) \varepsilon_{\kappa}^{\prime}\right] \delta_{n+11, v} \delta_{m u}}{(n+1)(n+2)(2 n+1)}, \\
& \bar{p}_{u v}^{m n}=\delta_{n v} \delta_{m u}+\frac{\left\{\left[\left(2 n^{2}+2 n+3\right) m^{2}+\left(2 n^{2}+2 n-3\right) n(n+1)\right] \bar{\varepsilon}_{r}^{\prime}+\left(4 n^{2}+4 n-3\right) m \varepsilon_{\kappa}^{\prime}\right\} \delta_{n v} \delta_{m u}}{n(n+1)(2 n-1)(2 n+3)} \\
& -\frac{(n+1)(n+m-1)(n+m) \bar{\varepsilon}_{r}^{\prime} \delta_{n-2, v} \delta_{m u}}{(n-1)(2 n-1)(2 n+1)}-\frac{n(n-m+1)(n-m+2) \bar{\varepsilon}_{r}^{\prime} \delta_{n+2, v} \delta_{m u}}{(n+2)(2 n+1)(2 n+3)}, \\
& \bar{q}_{u v}^{m n}=-\frac{\left[\left(n^{2}+n-3 m^{2}\right) \bar{\varepsilon}_{r}^{\prime}-m(2 n-1)(2 n+3) \varepsilon_{\kappa}^{\prime}\right] \delta_{n v} \delta_{m u}}{(2 n-1)(2 n+3)}+\frac{(n+1)(n+m-1)(n+m) \bar{\varepsilon}_{r}^{\prime} \delta_{n-2, v} \delta_{m u}}{(2 n-1)(2 n+1)} \\
& -\frac{n(n-m+1)(n-m+2) \bar{\varepsilon}_{r}^{\prime} \delta_{n+2, v} \delta_{m u}}{(2 n+1)(2 n+3)} \\
& \tilde{g}_{u v}^{m n}=\delta_{n v} \delta_{m u}+\frac{\left[\left(n^{2}+n-m^{2}\right) \bar{\mu}_{r}^{\prime}+m \mu_{\kappa}^{\prime}\right] \delta_{n v} \delta_{m u}}{n(n+1)}, \\
& \widetilde{e}_{u v}^{m n}=\frac{i(n+m)\left[m \bar{\mu}_{r}^{\prime}-(n+1) \mu_{\kappa}^{\prime}\right] \delta_{n-1, v} \delta_{m u}}{n(2 n+1)}+\frac{i(n-m+1)\left[m \bar{\mu}_{r}^{\prime}+n \mu_{\kappa}^{\prime}\right] \delta_{n+11, v} \delta_{m u}}{(n+1)(2 n+1)},
\end{aligned}
$$




$$
\begin{aligned}
\tilde{f}_{u v}^{m n}= & \frac{-i(n+m)\left[m \bar{\mu}_{r}^{\prime}-(n+1) \mu_{\kappa}^{\prime}\right] \delta_{n-1, v} \delta_{m u}}{(2 n+1)}+\frac{i(n-m+1)\left[m \bar{\mu}_{r}^{\prime}+n \mu_{\kappa}^{\prime}\right] \delta_{n+11, v} \delta_{m u}}{(2 n+1)}, \\
\bar{g}_{u v}^{m n}= & -\frac{i(n+m)(n+1)\left[m \bar{\mu}_{r}^{\prime}+(n-1) \mu_{\kappa}^{\prime}\right] \delta_{n-1, v} \delta_{m u}}{n(n-1)(2 n+1)}-\frac{i(n-m+1) n\left[m \bar{\mu}_{r}^{\prime}-(n+2) \mu_{\kappa}^{\prime}\right] \delta_{n+11, v} \delta_{m u}}{(n+1)(n+2)(2 n+1)}, \\
\bar{e}_{u v}^{m n}= & \delta_{n v} \delta_{m u}+\frac{\left\{\left[\left(2 n^{2}+2 n+3\right) m^{2}+\left(2 n^{2}+2 n-3\right) n(n+1)\right] \bar{\mu}_{r}^{\prime}+\left(4 n^{2}+4 n-3\right) m \mu_{\kappa}^{\prime}\right\} \delta_{n v} \delta_{m u}}{n(n+1)(2 n-1)(2 n+3)} \\
& -\frac{(n+1)(n+m-1)(n+m) \bar{\mu}_{r}^{\prime} \delta_{n-2, v} \delta_{m u}}{(n-1)(2 n-1)(2 n+1)}-\frac{n(n-m+1)(n-m+2) \bar{\mu}_{r}^{\prime} \delta_{n+2, v} \delta_{m u}}{(n+2)(2 n+1)(2 n+3)}, \\
\bar{f}_{u v}^{m n}= & -\frac{\left[\left(n^{2}+n-3 m^{2}\right) \bar{\mu}_{r}^{\prime}-m(2 n-1)(2 n+3) \mu_{\kappa}^{\prime}\right] \delta_{n v} \delta_{m u}}{(2 n-1)(2 n+3)}+\frac{(n+1)(n+m-1)(n+m) \bar{\mu}_{r}^{\prime} \delta_{n-2, v} \delta_{m u}}{(2 n-1)(2 n+1)} \\
& -\frac{n(n-m+1)(n-m+2) \bar{\mu}_{r}^{\prime} \delta_{n+2, v} \delta_{m u}}{(2 n+1)(2 n+3)} .
\end{aligned}
$$

Here, all the coefficients are obtained using the orthogonality of the VSWFs [32, 41, 42].

Therefore, one has

$$
\begin{aligned}
& \mu_{s} \bar{\mu}^{-1} \cdot \mathbf{B} \\
& =\sum_{n, m} \bar{E}_{m n}\left[\bar{d}_{m n} \mathbf{M}_{m n}^{(1)}(k, \mathbf{r})+\bar{c}_{m n} \mathbf{N}_{m n}^{(1)}(k, \mathbf{r})+\bar{w}_{m n} \mathbf{L}_{m n}^{(1)}(k, \mathbf{r})\right] \\
& \quad+\bar{w}_{00} \mathbf{L}_{00}^{(1)}(k, \mathbf{r}),
\end{aligned}
$$

where

$$
\begin{gathered}
\bar{d}_{m n}=\sum_{v, u} \frac{\bar{E}_{u v}}{\bar{E}_{m n}}\left[\tilde{g}_{m n}^{u v} d_{u v}+\bar{g}_{m n}^{u v} c_{u v}\right], \\
\bar{c}_{m n}=\sum_{v, u} \frac{\bar{E}_{u v}}{\bar{E}_{m n}}\left[\tilde{e}_{m n}^{u v} d_{u v}+\bar{e}_{m n}^{u v} c_{u v}\right], \\
\bar{w}_{m n}=\sum_{v, u} \frac{\bar{E}_{u v}}{\bar{E}_{m n}}\left[\tilde{f}_{m n}^{u v} d_{u v}+\bar{f}_{m n}^{u v} c_{u v}\right], \\
\bar{w}_{00}=-\left[\sqrt{\frac{2}{3}} \mu_{\kappa}^{\prime} d_{01}+\sqrt{\frac{2}{15}} \bar{\mu}_{r}^{\prime} c_{02}\right] E_{0} .
\end{gathered}
$$

Repeat the procedure and reuse the properties of the VSWFs bellow until (31) can be written in the form of $\mathbf{M}_{m n}^{(1)}(k, \mathbf{r})$, $\mathbf{N}_{m n}^{(1)}(k, \mathbf{r})$, and $\mathbf{L}_{m n}^{(1)}(k, \mathbf{r})$ :

$$
\begin{gathered}
\nabla \times \mathbf{M}_{m n}^{(j)}-k \mathbf{N}_{m n}^{(j)}=0, \\
\nabla \times \mathbf{N}_{m n}^{(j)}-k^{2} \mathbf{M}_{m n}^{(j)}=0, \\
\nabla \times \mathbf{L}_{m n}^{(j)}=0 .
\end{gathered}
$$

One gets

$$
\sum_{n, m} \bar{E}_{m n}\left[\tilde{\tilde{d}}_{m n} \mathbf{M}_{m n}^{(1)}(k, \mathbf{r})+\widetilde{\tilde{c}}_{m n} \mathbf{N}_{m n}^{(1)}(k, \mathbf{r})\right]=0
$$

with

$$
\begin{aligned}
& \tilde{\tilde{d}}_{m n}=k^{2} \sum_{v, u} \frac{\bar{E}_{u v}}{\bar{E}_{m n}}\left[\bar{p}_{m n}^{u v} \bar{d}_{u v}+\tilde{p}_{m n}^{u v} \bar{c}_{u v}\right]-k_{s}^{2} d_{m n}, \\
& \tilde{\widetilde{c}}_{m n}=k^{2} \sum_{v, u} \frac{\bar{E}_{u v}}{\bar{E}_{m n}}\left[\bar{o}_{m n}^{u v} \bar{d}_{u v}+\tilde{o}_{m n}^{u v} \bar{c}_{u v}\right]-k_{s}^{2} c_{m n} .
\end{aligned}
$$

Then, we write the equation form:

$$
\left(\begin{array}{cc}
\widetilde{P} & \bar{P} \\
\widetilde{O} & \bar{O}
\end{array}\right)\left(\begin{array}{l}
d \\
c
\end{array}\right)=\lambda\left(\begin{array}{l}
d \\
c
\end{array}\right)
$$

The expression for $\widetilde{P}, \bar{P}, \widetilde{O}, \bar{O}$ is in [32]. Let $\lambda_{l}$ and $\left(d_{m n, l}, c_{m n, l}\right)^{T}$, denote, respectively, the eigenvalues and the corresponding eigenvectors of eigensystem (40), with $l$ representing the index of eigenvalues and corresponding eigenvectors. One can then build a new set of vector $\mathbf{V}_{l}$ in space based on the eigenvectors:

$$
\mathbf{V}_{l}=-\frac{k_{l}}{\omega} \sum_{n, m} \bar{E}_{m n}\left[d_{m n, l} \mathbf{M}_{m n}^{(1)}\left(k_{l}, \mathbf{r}\right)+c_{m n, l} \mathbf{N}_{m n}^{(1)}\left(k_{l}, \mathbf{r}\right)\right]
$$

Thus, we can express $\mathbf{B}$ as

$$
\begin{aligned}
\mathbf{B} & =\sum_{l} \alpha_{l} \mathbf{V}_{l} \\
& =-\sum_{l} \alpha_{l} \frac{k_{l}}{\omega} \sum_{n, m} \bar{E}_{m n}\left[d_{m n, l} \mathbf{M}_{m n}^{(1)}\left(k_{l}, \mathbf{r}\right)+c_{m n, l} \mathbf{N}_{m n}^{(1)}\left(k_{l}, \mathbf{r}\right)\right]
\end{aligned}
$$


Use the relation of (6), $\mathbf{H}$ and $\mathbf{E}$ fields can be written as

$$
\begin{aligned}
\mathbf{H}= & \bar{\mu}^{-1} \cdot \mathbf{B} \\
= & -\sum_{n, m} \bar{E}_{m n} \sum_{l} \frac{k_{l}}{\omega \mu_{s}} \alpha_{l} \\
& \times\left[\bar{d}_{m n, l} \mathbf{M}_{m n}^{(1)}\left(k_{l}, \mathbf{r}\right)+\bar{c}_{m n, l} \mathbf{N}_{m n}^{(1)}\left(k_{l}, \mathbf{r}\right)+w_{m n, l} \mathbf{L}_{m n}^{(1)}\left(k_{l}, \mathbf{r}\right)\right] \\
& -\sum_{l} \frac{k_{l}}{\omega \mu_{s}} \alpha_{l}\left[w_{00, l} \mathbf{L}_{00}^{(1)}(k, \mathbf{r})\right], \\
\mathbf{E}= & -i \sum_{n, m} \bar{E}_{m n} \sum_{l} \alpha_{l}\left[c_{m n, l} \mathbf{M}_{m n}^{(1)}\left(k_{l}, \mathbf{r}\right)+d_{m n, l} \mathbf{N}_{m n}^{(1)}\left(k_{l}, \mathbf{r}\right)\right. \\
& \left.\quad+\frac{w_{m n, l}}{\lambda_{l}} \mathbf{L}_{m n}^{(1)}\left(k_{l}, \mathbf{r}\right)\right]-i \sum_{l} \alpha_{l}\left[\frac{\overline{\bar{w}}_{00, l}}{\lambda_{l}} \mathbf{L}_{00}^{(1)}(k, \mathbf{r})\right] .
\end{aligned}
$$

As we have discussed in the angular expansion method, here, we also give the form of the incident wave and scatted wave.

For incident wave,

$$
\begin{aligned}
\mathbf{E}_{\mathrm{inc}} & =-\sum_{n, m} i \bar{E}_{m n}\left[p_{m n} \mathbf{N}_{m n}^{(1)}(k, \mathbf{r})+q_{m n} \mathbf{M}_{m n}^{(1)}(k, \mathbf{r})\right], \\
\mathbf{H}_{\mathrm{inc}} & =-\frac{k_{0}}{\omega \mu_{0}} \sum_{n, m} \bar{E}_{m n}\left[q_{m n} \mathbf{N}_{m n}^{(1)}(k, \mathbf{r})+p_{m n} \mathbf{M}_{m n}^{(1)}(k, \mathbf{r})\right] .
\end{aligned}
$$

For scattered wave,

$$
\begin{aligned}
\mathbf{E}_{s} & =\sum_{n, m} i \bar{E}_{m n}\left[a_{m n} \mathbf{N}_{m n}^{(3)}\left(k_{0}, \mathbf{r}\right)+b_{m n} \mathbf{M}_{m n}^{(3)}\left(k_{0}, \mathbf{r}\right)\right] \\
\mathbf{H}_{s} & =\frac{k_{0}}{\omega \mu_{0}} \sum_{n, m} \bar{E}_{m n}\left[b_{m n} \mathbf{N}_{m n}^{(3)}\left(k_{0}, \mathbf{r}\right)+a_{m n} \mathbf{M}_{m n}^{(3)}\left(k_{0}, \mathbf{r}\right)\right] .
\end{aligned}
$$

The relevant coefficients are in [41]. Then, match boundary conditions on the sphere surface, we can get a system of equations for the coefficients, which is

$$
\begin{gathered}
{\left[\frac{\xi_{n}^{\prime}(x)}{\psi_{n}^{\prime}(x)}\right] a_{m n}+\sum_{l}\left[\frac{1}{m_{s} \bar{k}_{l}} \frac{\psi_{n}^{\prime}\left(\bar{k}_{l} m_{s} x\right)}{\psi_{n}^{\prime}(x)} d_{m n, l}\right] \alpha_{l}} \\
+\sum_{l}\left[\frac{\mu_{0}}{\mu_{s}} \frac{j_{n}\left(\bar{k}_{l} m_{s} x\right)}{\psi_{n}^{\prime}(x)} \omega_{m n, l}\right] \alpha_{l}=p_{m n}, \\
{\left[\frac{\xi_{n}(x)}{\psi_{n}(x)}\right] b_{m n}+\sum_{l}\left[\frac{1}{m_{s} \bar{k}_{l}} \frac{\psi_{n}\left(\bar{k}_{l} m_{s} x\right)}{\psi_{n}(x)} c_{m n, l}\right] \alpha_{l}=q_{m n},} \\
{\left[\frac{\xi_{n}(x)}{\psi_{n}(x)}\right] a_{m n}+\sum_{l}\left[\frac{\mu_{0} \lambda_{l}}{\mu_{s}} \frac{\psi_{n}\left(\bar{k}_{l} m_{s} x\right)}{\psi_{n}(x)} d_{m n, l}\right] \alpha_{l}=p_{m n},} \\
{\left[\frac{\xi_{n}^{\prime}(x)}{\psi_{n}^{\prime}(x)}\right] b_{m n}+\sum_{l}\left[\frac{\mu_{0} \lambda_{l}}{\mu_{s}} \frac{\psi_{n}^{\prime}\left(\bar{k}_{l} m_{s} x\right)}{\psi_{n}^{\prime}(x)} c_{m n, l}\right] \alpha_{l}} \\
+\sum_{l}\left[\frac{\mu_{0}}{\mu_{s}} \frac{j_{n}\left(\bar{k}_{l} m_{s} x\right)}{\psi_{n}^{\prime}(x)} \omega_{m n, l}\right] \alpha_{l}=q_{m n},
\end{gathered}
$$

then rewrite the matrix in a clear form:

$$
\begin{aligned}
& \left(\begin{array}{ll}
\Lambda & 0 \\
0 & \bar{\Lambda}
\end{array}\right)\left(\begin{array}{l}
a \\
b
\end{array}\right)+\left(\begin{array}{l}
V \\
\bar{V}
\end{array}\right) \tilde{\alpha}=\left(\begin{array}{l}
p \\
q
\end{array}\right), \\
& \left(\begin{array}{ll}
\bar{\Lambda} & 0 \\
0 & \Lambda
\end{array}\right)\left(\begin{array}{l}
a \\
b
\end{array}\right)+\left(\begin{array}{l}
\bar{U} \\
U
\end{array}\right) \tilde{\alpha}=\left(\begin{array}{l}
p \\
q
\end{array}\right)
\end{aligned}
$$

with

$$
\begin{aligned}
\Lambda_{m n, u v} & =S_{n}(x) \delta_{n v} \delta_{m u}, \\
\bar{\Lambda}_{m n, u v} & =\bar{S}_{n}(x) \delta_{n v} \delta_{m u}, \\
U_{m n, l} & =\frac{1}{m_{s} \bar{k}_{l}} \bar{T}_{n}\left(x, m_{s} \bar{k}_{l} x\right) c_{m n, l}, \\
\bar{U}_{m n, l} & =\frac{1}{m_{s} \bar{k}_{l}} \bar{T}_{n}\left(x, m_{s} \bar{k}_{l} x\right) d_{m n, l}, \\
V_{m n, l} & =\frac{\mu_{0} \lambda_{l}}{\mu_{s}} T_{n}\left(x, m_{s} \bar{k}_{l} x\right) d_{m n, l}, \\
\bar{V}_{m n, l} & =\frac{\mu_{0} \lambda_{l} \bar{T}_{n}\left(x, m_{s} \bar{k}_{l} x\right) c_{m n, l}+W_{m n, l},}{\mu_{s}} \quad \bar{T}_{n}\left(x, m_{s} \bar{k}_{l} x\right) \\
W_{m n, l} & =\frac{\mu_{0}}{\mu_{s}} \frac{1}{m_{s} \bar{k}_{l} x} \frac{D_{n}^{(1)}\left(m_{s} \bar{k}_{l} x\right)}{D_{m n, l},} \\
S_{n}(x) & =\frac{\xi_{n}(x)}{\psi_{n}(x)} \quad \bar{S}_{n}(x)=\frac{\xi_{n}^{\prime}(x)}{\psi_{n}^{\prime}(x)}, \\
T_{n}(x, z) & =\frac{\psi_{n}(z)}{\psi_{n}(x)} \quad \bar{T}_{n}(x, z)=\frac{\psi_{n}^{\prime}(z)}{\psi_{n}^{\prime}(x)} .
\end{aligned}
$$

Afterwards, we can solve the equations and get the coefficients matrix, and the scattering information is in the matrix $\left(\begin{array}{l}a \\ b\end{array}\right)$ :

$$
\begin{aligned}
\tilde{\alpha} & =R\left(\begin{array}{l}
p \\
q
\end{array}\right), \\
\left(\begin{array}{l}
a \\
b
\end{array}\right) & =S\left(\begin{array}{l}
p \\
q
\end{array}\right),
\end{aligned}
$$

where

$$
\begin{gathered}
Z=\left(\begin{array}{cc}
Y & 0 \\
0 & -Y
\end{array}\right)^{-1}\left(\begin{array}{c}
V-\bar{U} \\
\bar{V}-U
\end{array}\right), \\
S=Z R \\
Y=\bar{\Lambda}-\Lambda .
\end{gathered}
$$

2.4. Numerical Results and Comparison. For the two methods mentioned above, difference between them will be analyzed as follows. In angular expansion method, the plane wave expansion is employed and the wave in such complex medium is a combination of plane wave of different direction. The whole procedure is clear and easy to understand. 
At the same time, the wave number $k$ has its physical meaning, which can be expressed. Theoretically, this method can deal with any complex medium. Different from angular expansion method, the T-matrix method does not give an expression for the wave number, the properties of spherical vector wave function are used instead and all the wave information is gathered from the matrix. In computation, the T-matrix method has more advantage over angular expansion method, which does not need to consider grid and can be applied to calculate larger problem.

As many discussions and comparisons published already, here we mainly show the strength of the two methods [32]. Figure 1 shows the gyroelectric influence on the Radar cross-section, here $\mu_{\kappa}=0, \quad \mu_{s} \mu_{r}=4 \mu_{0} \mu_{s}=2 \mu_{0}, \quad \varepsilon_{s} \varepsilon_{r}=$ $4 \varepsilon_{0}, \quad \varepsilon_{s}=2 \varepsilon_{0}$. As we can see, when the off-diagonal parameters increase, the backscattering $\left(180^{\circ}\right)$ will also increase. Compared to Figure 1, Figure 2 shows the gyromagnetic influence on the RCS, here $\varepsilon_{s} \varepsilon_{r}=2.4 \varepsilon_{0}, \varepsilon_{s}=2 \varepsilon_{0}$, the less scattering areas is reduced from three to one. Figure 3 gives a general case, the influences of permittivity and permeability are both considered; and this is also a lossy sphere, where $\varepsilon_{s}=(2+1.0 i) \varepsilon_{0}, \mu_{s}=(2+1.0 i) \mu_{0}$. When increasing the permittivity elements of the materials, the scattering for larger than $80^{\circ}$ area will increase obviously.

\section{Electromagnetic Wave Scattering by a Radial Anisotropic Sphere}

3.1. Radial Anisotropy of Spheres. As another type medium, the radial anisotropy materials have parameters given by [15]

$$
\bar{\varepsilon}=\left(\begin{array}{ccc}
\varepsilon_{r r} & 0 & 0 \\
0 & \tau & \sigma \\
0 & -\sigma & \tau
\end{array}\right), \quad \mu=\left(\begin{array}{ccc}
\mu_{r r} & 0 & 0 \\
0 & \gamma & \xi \\
0 & -\xi & \gamma
\end{array}\right) \text {, }
$$

where the identity dyadic is expressed as $\widehat{\mathbf{I}}=\widehat{\mathbf{r}} \widehat{\mathbf{r}}+\hat{\boldsymbol{\theta}} \hat{\boldsymbol{\theta}}+\hat{\boldsymbol{\phi}} \hat{\boldsymbol{\phi}}$. Different from the Cartesian anisotropic (CA), less discussion have been published, because the radial anisotropic (RA) material is difficult to be fabricated [7]. The research attracts much attention recently due to the properties of materials with novel parameters. How RA particles interact with waves is an essential topic which will provide us a more physical insight into the invisibility phenomenon [43-47], enhanced surface plasmon resonance $[48,49]$, and Fano resonance [50]. Although Monzon developed the variables separation method and demonstrated a perfect explanation to the solution form [15], researchers prefer the Debye potential or other potentials similar [51-53]. Recently, Novitsky has stated a general form for the material, and then extend the T-matrix method to a multilayer case [54]. When a Hamilton operator is used, the Maxwell equations will be greatly simplified. By employing the impedance matrix, the field area is both simple and clear. However, this solution is just another form of variables separation method and only suitable to the uniaxial anisotropic case, which does not satisfy the general situation [54]. Herein, we explain Monzon's method and conclude an explanation to the solution.

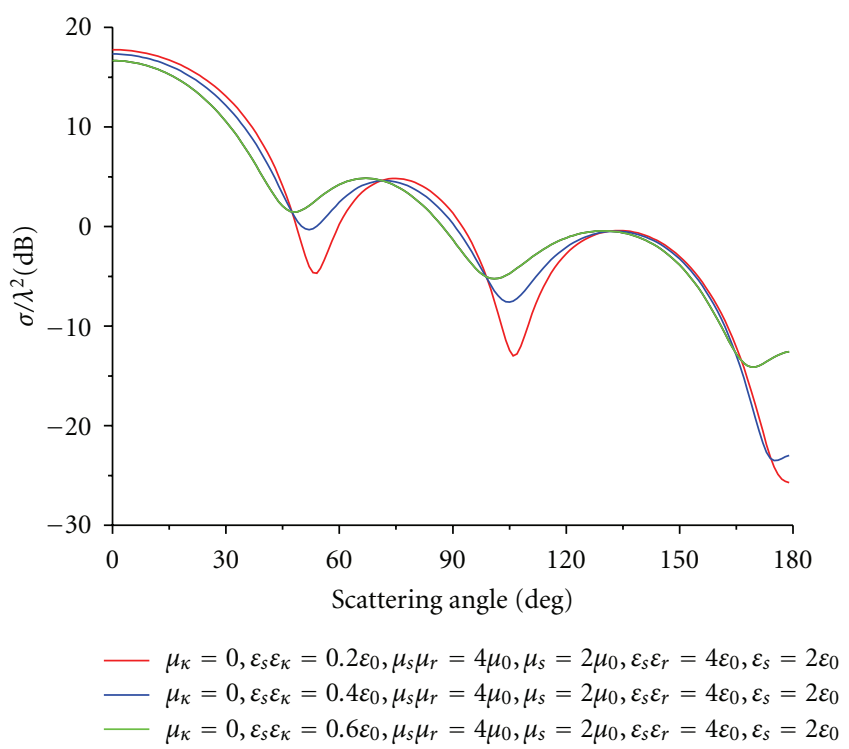

Figure 1: Normalized Radar cross-section (RCS) values versus the scattering angle. Different gyroelectric influence is considered. Here, $k_{0} r=\pi$.

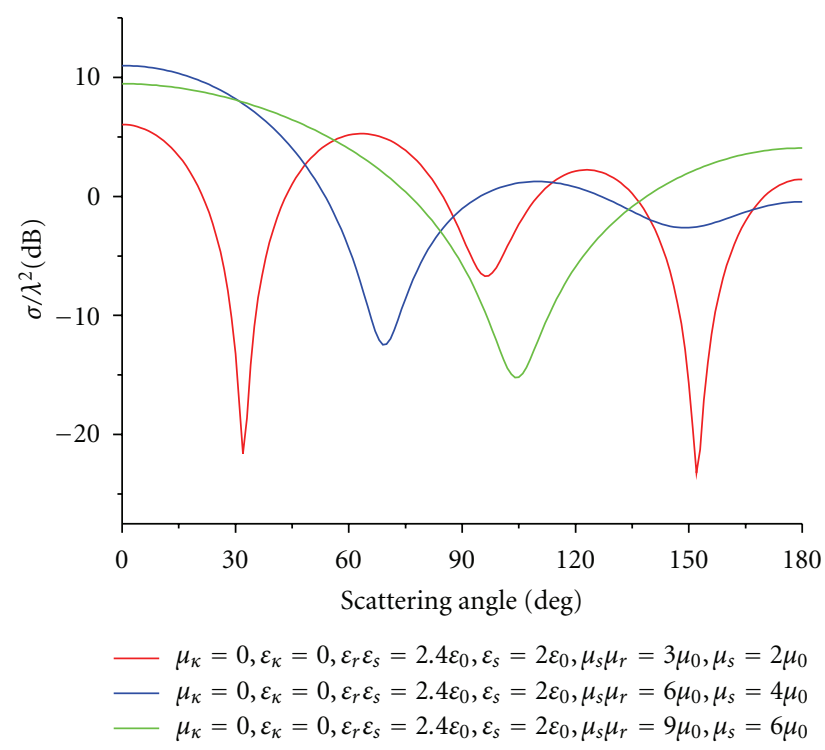

Figure 2: Normalized Radar cross-section (RCS) values versus the scattering angle. Different gyromagnetic influence is considered. Here, $k_{0} r=0.75 \pi$.

3.2. Variables Separation Method. Firstly, we define new vectors to be [15]

$$
\widehat{\boldsymbol{\xi}}_{ \pm}=\frac{\hat{\boldsymbol{\theta}} \pm j \hat{\boldsymbol{\phi}}}{\sqrt{2} \sin \theta} .
$$




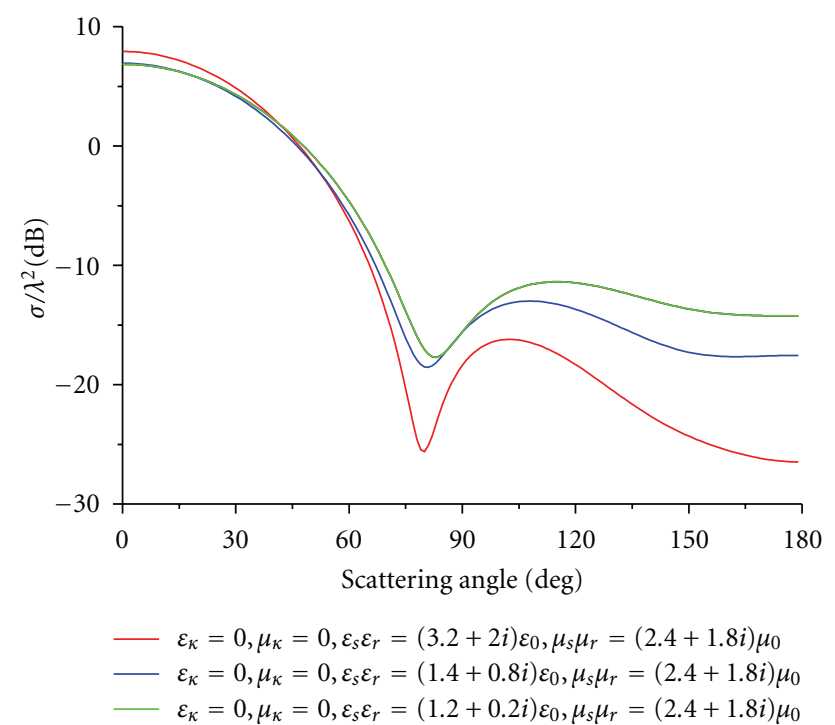

Figure 3: Normalized Radar Cross Section (RCS) values versus the scattering angle. Here the permeability and the permittivity are complex number. The Loss gyrotropic influence is considered. Here $k_{0} r=0.75 \pi$.

Then, the E-field and $\mathbf{H}$-field can be expressed in the new coordinates:

$$
\begin{aligned}
& \mathbf{E}(\mathbf{r})=E_{r} \hat{\mathbf{r}}+e_{-} \hat{\boldsymbol{\xi}}_{+}+e_{+} \overline{\boldsymbol{\xi}}_{-} \quad e_{ \pm}=\frac{\sin \theta}{\sqrt{2}}\left(E_{\theta} \pm j E_{\phi}\right), \\
& \mathbf{H}(\mathbf{r})=H_{r} \hat{\mathbf{r}}+h_{-} \hat{\boldsymbol{\xi}}_{+}+h_{+} \overline{\boldsymbol{\xi}}_{-} \quad h_{ \pm}=\frac{\sin \theta}{\sqrt{2}}\left(H_{\theta} \pm j H_{\phi}\right),
\end{aligned}
$$

Similarly, the displacement vector is provided:

$$
\begin{aligned}
& \bar{\varepsilon} \cdot \mathbf{E}=\varepsilon_{r r} E_{r} \hat{\mathbf{r}}+{ }_{+} \alpha_{+} e_{-} \overline{\boldsymbol{\xi}}_{+}+\alpha_{-} e_{+} \overline{\boldsymbol{\xi}}_{-}, \quad \alpha_{ \pm}=\tau \pm j \sigma, \\
& \bar{\mu} \cdot \mathbf{H}=\mu_{r r} H_{r} \hat{\mathbf{r}}+{ }_{+} \beta_{+} h_{-} \overline{\boldsymbol{\xi}}_{+}+\beta_{-} h_{+} \overline{\boldsymbol{\xi}}_{-}, \quad \beta_{ \pm}=\gamma \pm j \zeta .
\end{aligned}
$$

Here, two operators are defined as

$$
D_{ \pm}=\frac{1}{\sqrt{2} r}\left(\sin \theta \frac{\partial}{\partial \theta} \pm j \frac{\partial}{\partial \phi}\right), \quad D_{r}=\frac{1}{r} \frac{\partial}{\partial r} \cdot r,
$$

then the Laplace operator can be written as

$$
\nabla=r \frac{\partial}{\partial r}+\widehat{\xi}_{+} D_{-}+\hat{\xi}_{-} D_{+},
$$

and Maxwell equations is correspondingly rewritten as

$$
\begin{aligned}
\nabla \times \mathbf{E}= & \frac{j \widehat{\mathbf{r}}}{\sin ^{2} \theta}\left\{D_{+} e_{-}-D_{-} e_{+}\right\}+j \bar{\xi}_{+}\left\{D_{-} E_{r}-D_{r} e_{-}\right\} \\
& +j \bar{\xi}_{-}\left\{D_{r} e_{+}-D_{+} E_{r}\right\}, \\
\nabla \times \mathbf{H}= & \frac{j \widehat{\mathbf{r}}}{\sin ^{2} \theta}\left\{D_{+} h_{-}-D_{-} h_{+}\right\}+j \bar{\xi}_{+}\left\{D_{-} H_{r}-D_{r} h_{-}\right\} \\
& +j \bar{\xi}_{-}\left\{D_{r} h_{+}-D_{+} H_{r}\right\},
\end{aligned}
$$

we insert (54) and (57) into (2) and (3). After some calculations including repeated operations on Maxwell's equations, a coupled set of differential equations involving only the radial field components can be obtained [15]:

$$
\begin{gathered}
\left\{\frac{\mu_{r r}}{\gamma r} D_{r}^{2}\left(r H_{r}\right)+\frac{2}{\sin ^{2} \theta} D_{+} D_{-} H_{r}+\frac{\omega^{2}}{\tau} \mu_{r r}\left(\tau^{2}+\sigma^{2}\right) H_{r}\right\} \\
+\frac{j \omega \varepsilon_{r r}}{\gamma \tau r}(\sigma \gamma+\tau \zeta) D_{r}\left(r E_{r}\right)=0, \\
\left\{\frac{\varepsilon_{r r}}{\tau r} D_{r}^{2}\left(r E_{r}\right)+\frac{2}{\sin ^{2} \theta} D_{+} D_{-} E_{r}+\frac{\omega^{2}}{\tau} \varepsilon_{r r}\left(\gamma^{2}+\zeta^{2}\right) E_{r}\right\} \\
-\frac{j \omega \mu_{r r}}{\gamma \tau r}(\sigma \gamma+\tau \zeta) D_{r}\left(r H_{r}\right)=0,
\end{gathered}
$$

where

$$
D_{+} D_{-}=\frac{1}{2 r^{2}}\left[\frac{\partial^{2}}{\partial \phi^{2}}+\sin \theta \frac{\partial}{\partial \theta} \sin \theta \frac{\partial}{\partial \theta}\right] .
$$

The field can be expressed in the following form:

$$
\begin{aligned}
& E_{r}=\sum_{n, m} E_{m n}(r) P_{n}^{m}(\cos \theta) e^{j m \phi}, \\
& H_{r}=\sum_{n, m} H_{m n}(r) P_{n}^{m}(\cos \theta) e^{j m \phi} .
\end{aligned}
$$

Also when $D_{+} D_{-}$operate on other components, it has the following properties:

$$
D_{+} D_{-}=-n(n+1) \frac{\sin ^{2} \theta}{2 r^{2}}
$$

we write the (58) in a convenient form:

$$
d^{2} f+\alpha_{1}(\rho) f-\delta d g=0 \quad d^{2} g+\alpha_{2}(\rho) g-\delta d f=0
$$

with

$$
\begin{array}{cc}
d=\frac{d}{d \rho} \quad \rho=\kappa r \quad \kappa=\omega \sqrt{\varepsilon_{r r} \mu_{r r}} \quad \delta=\frac{(\sigma \gamma+\tau \zeta)}{\sqrt{\tau \gamma \varepsilon_{r r} \mu_{r r}}}, \\
\alpha_{1}(\rho)=A_{1}-\frac{B_{1}}{\rho^{2}} \quad \alpha_{2}(\rho)=A_{2}-\frac{B_{2}}{\rho^{2}} \\
A_{1}=\frac{\tau}{\gamma} \frac{\left(\gamma^{2}+\zeta^{2}\right)}{\varepsilon_{r r} \mu_{r r}}, & A_{2}=\frac{\gamma}{\tau} \frac{\left(\tau^{2}+\sigma^{2}\right)}{\varepsilon_{r r} \mu_{r r}}, \\
B_{1}=\frac{\tau}{\varepsilon_{r r}} n(n+1), & B_{2}=\frac{\gamma}{\mu_{r r}} n(n+1) .
\end{array}
$$

For (62), there is an analytical solution to the differential equations only when the coupled component is 0 (the isotropic or uniaxial anisotropic case). For a general case $(\delta \neq 0)$, there is no analytical solution for this kind of differential equations in mathematics [55]. Therefore, we 
have to use the series to stand for the equation roots and the procedure is as follows:

$$
\begin{gathered}
f=\sum_{n=-\infty}^{\infty} \rho^{\lambda+n} K_{n}, \\
g=\sum_{n=-\infty}^{\infty} \rho^{\lambda+n} Q_{n}, \\
{\left[(\lambda+n)(\lambda+n-1)-B_{1}\right] K_{n}+A_{1} K_{n-2}=\delta(\lambda+n-1) Q_{n-1},} \\
{\left[(\lambda+n)(\lambda+n-1)-B_{2}\right] Q_{n}+A_{2} Q_{n-2}=\delta(\lambda+n-1) K_{n-1},} \\
Q_{1}=\frac{\delta(67)}{\left[2 \tau_{1}+1+\left(B_{1}-B_{2}\right)\right]}, \quad K_{2}=\frac{\delta\left(\tau_{1}+3 / 2\right) Q_{1}-A_{1}}{4\left(\tau_{1}+1\right)} .
\end{gathered}
$$

When the original condition is given, the coefficients can be calculated step by step and then an expression for the radial component as we needed from (66) and (67) can be achieved. If the off-diagonal parameters are zero, $E_{r}$ and $H_{r}$ are not coupled and we encounter the most discussed case [51]:

$$
\begin{gathered}
E_{r}=\frac{\omega}{i k_{t}^{2}}\left(\frac{\partial^{2}}{\partial r^{2}}+k_{t}^{2}\right) \Psi_{T M}, \\
E_{\theta}=\frac{\omega}{i k_{t}^{2}} \frac{1}{r} \frac{\partial^{2} \Psi_{T M}}{\partial r \partial \theta}-\frac{1}{\varepsilon_{0} \varepsilon_{t}} \frac{1}{r \sin \theta} \frac{\partial \Psi_{T E}}{\partial \phi}, \\
E_{\phi}=\frac{\omega}{i k_{t}^{2}} \frac{1}{r \sin \theta} \frac{\partial^{2} \Psi_{T M}}{\partial r \partial \phi}+\frac{1}{\varepsilon_{0} \varepsilon_{t}} \frac{1}{r} \frac{\partial \Psi_{T E}}{\partial \theta}, \\
H_{r}=\frac{\omega}{i k_{t}^{2}}\left(\frac{\partial^{2}}{\partial r^{2}}+k_{t}^{2}\right) \Psi_{T E} \\
H_{\theta}=\frac{1}{\mu_{0} \mu_{t}} \frac{1}{r \sin \theta} \frac{\partial \Psi_{T M}}{\partial \phi}+\frac{\omega}{i k_{t}^{2}} \frac{1}{r} \frac{\partial^{2} \Psi_{T E}}{\partial r \partial \theta} \\
H_{\phi}=-\frac{1}{\mu_{0} \mu_{t}} \frac{1}{r} \frac{\partial \Psi_{T M}}{\partial \theta}+\frac{\omega}{i k_{t}^{2}} \frac{1}{r \sin \theta} \frac{\partial^{2} \Psi_{T E}}{\partial r \partial \phi} .
\end{gathered}
$$

For a plane wave, there are many discussions already; here, we only show the result for the internal and external field:

$$
\begin{gathered}
a_{n}=\frac{\sqrt{\mu_{t} / \varepsilon_{t}} j_{n}\left(k_{0} a\right) j_{v_{1}}^{\prime}\left(k_{t} a\right)-j_{n}^{\prime}\left(k_{0} a\right) j_{v_{1}}\left(k_{t} a\right)}{h_{n}^{(2)^{\prime}}\left(k_{0} a\right) j_{v_{1}}\left(k_{t} a\right)-\sqrt{\mu_{t} / \varepsilon_{t}} h_{n}^{(2)}\left(k_{0} a\right) j_{v_{1}}^{\prime}\left(k_{t} a\right)} T_{n}, \\
b_{n}=\frac{\sqrt{\mu_{t} / \varepsilon_{t}} j_{n}^{\prime}\left(k_{0} a\right) j_{v_{2}}\left(k_{t} a\right)-j_{n}\left(k_{0} a\right) j_{v_{2}}^{\prime}\left(k_{t} a\right)}{h_{n}^{(2)}\left(k_{0} a\right) j_{v_{2}}^{\prime}\left(k_{t} a\right)-\sqrt{\mu_{t} / \varepsilon_{t}} h_{n}^{(2))^{\prime}}\left(k_{0} a\right) j_{v_{2}}\left(k_{t} a\right)} T_{n},
\end{gathered}
$$

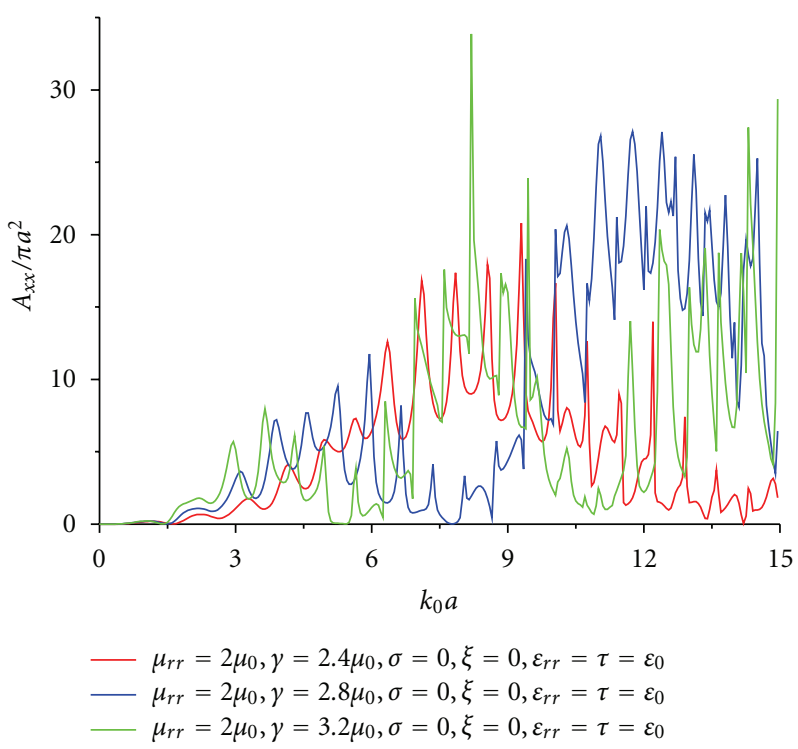

FIGURE 4: Normalized backscattering values versus sphere radius. Different negative anisotropic value influence is considered. Here, we increase the negative values.

$$
\begin{aligned}
c_{n} & =\frac{i}{\sqrt{\mu_{t} / \varepsilon_{t}} h_{n}^{(2)}\left(k_{0} a\right) j_{v_{1}}^{\prime}\left(k_{t} a\right)-h_{n}^{(2)^{\prime}}\left(k_{0} a\right) j_{v_{1}}\left(k_{t} a\right)} T_{n}, \\
d_{n} & =\frac{i \sqrt{\mu_{t} / \varepsilon_{t}}}{h_{n}^{(2)}\left(k_{0} a\right) j_{v_{2}}^{\prime}\left(k_{t} a\right)-\sqrt{\mu_{t} / \varepsilon_{t}} h_{n}^{(2)^{\prime}}\left(k_{0} a\right) j_{v_{2}}\left(k_{t} a\right)} T_{n}, \\
T_{n} & =\frac{i^{-n}(2 n+1)}{n(n+1)} .
\end{aligned}
$$

Using these coefficients, there are many interesting results [7], which will not be discussed here.

3.3. Numerical Results. Results of the method using the above coefficients are presented in the following. Figure 4 states the negative anisotropy $\left(\mu_{r r}<\gamma\right)$ influence on the backscattering when $\varepsilon_{r r}=\tau=\varepsilon_{0}$. Sharp changes can be observed when the radius changes. Compared to Figure 4, Figure 5 shows the positive anisotropy $\left(\mu_{r r}>\gamma\right)$ influence on the backscattering, from which the oscillations are more regular. Figures 6 and 7 give a general case for the absorbing sphere. Taking the influence of negative absorbing into accounting, we can see from Figure 6 that the backscattering will approach to a certain value as the radius increases. There will be more backscattering as the sphere has much loss. The positive absorbing properties are discussed in Figure 7, which behaves a similar phenomenon with Figure 6.

\section{Discussion and Conclusion}

In this review, two different types of anisotropy have been discussed in Cartesian and spherical coordinates, respectively. The role of anisotropy in scattering properties is 


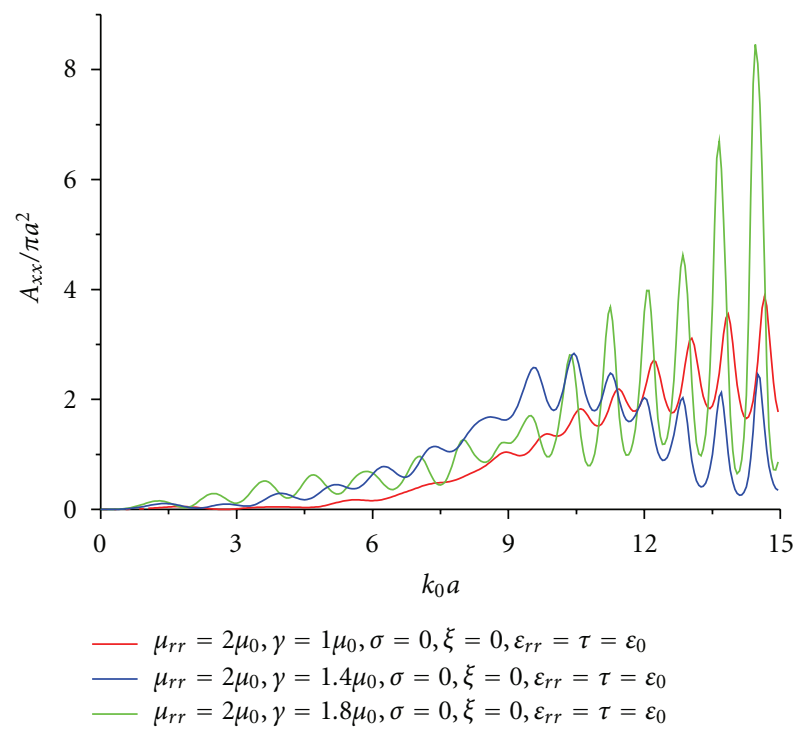

Figure 5: Normalized backscattering values versus sphere radius. Different positive anisotropic value influence is considered. Here, we reduce the positive values.

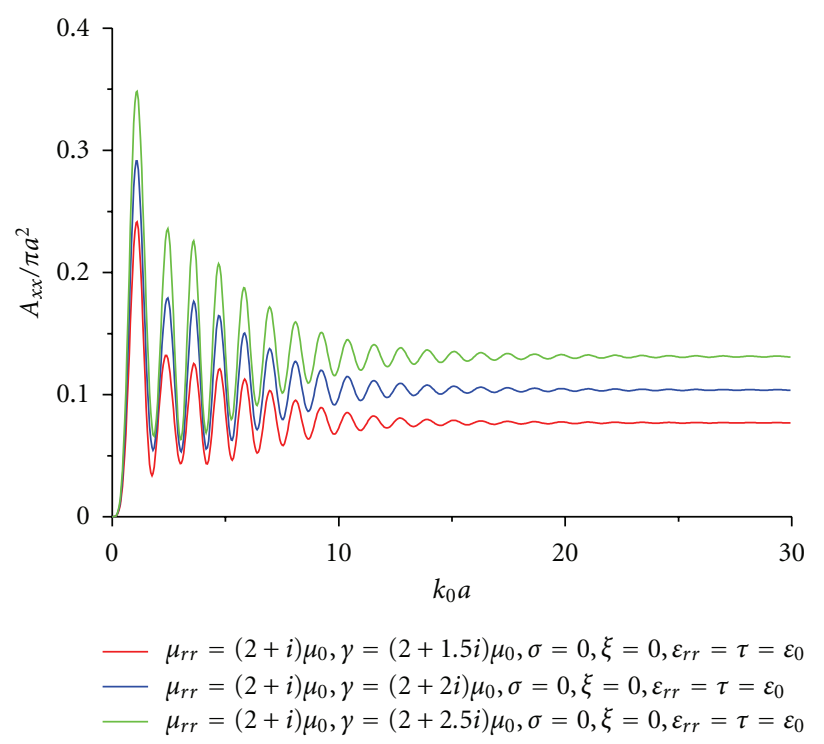

FIGURE 6: Normalized backscattering values versus sphere radius. Lossy negative anisotropic influence is considered. Here, the imaginary part for the permeability is bigger than 1 .

characterized analytically. Two main methods are introduced in Cartesian coordinates. As the first one, the angular expansion method can be used in the infinitely large area and present an obvious explanation for the properties of the medium. Different from the first method, the Tmatrix method can avoid the complex function by using the orthogonal properties for $\mathbf{M}_{m n}^{(l)}, \mathbf{N}_{m n}^{(l)}$, and $\mathbf{L}_{m n}^{(l)}$. There is no need for researchers to consider the mesh before the calculation. Since these two methods are analytical and semianalytical, respectively, the parameter's influence on

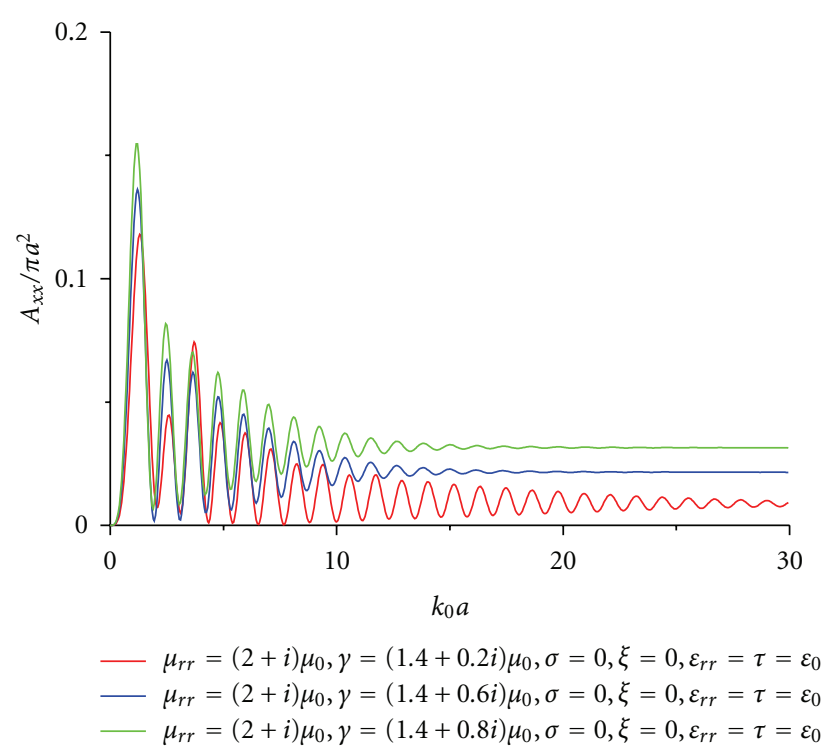

Figure 7: Normalized backscattering values versus sphere radius. Lossy positive anisotropic influence is considered. Here, the imaginary part for the permeability is smaller than 1 .

every part is very clear and thus gives us an insight into anisotropy materials.

For a spherical coordinate anisotropic material, no matter which operators are used, the variables separation remains the main method to settle the problem. Though the Debye potentials can be widely used based on the properties of the Maxwell equations, it is still not suitable to solve the coupled gyrotropic materials. Monzon's work demonstrated the reason why and how the electric field and magnetic field coupled. When a specific situation is considered, for example, a uniaxial anisotropic media, the electric field and the magnetic field can be separated and be both expressed in the radial function form. This review provides the convenience for researchers who want to explore new properties of anisotropic materials and, therefore, design new structures.

\section{Acknowledgments}

The authors wish to thank Professor Li Lewei, and Ms. Ong Weeling for helpful discussions. This work is supported by CSC scholarship and Key Program of NSFC-Guangdong Union Foundation under Grant no. U0835004.

\section{References}

[1] L. Lorentz, "Lysbevaegelsen i og uden for en haf plane lysbolger belyst kulge," Videnskab Selskab Skrifter, vol. 6, pp. 1-62, 1890.

[2] G. Mie, "Beiträge zur optik trüber medien, speziell kolloidaler metallösungen," Annalen der Physik, vol. 25, no. 3, pp. 377445, 1908.

[3] B. S. Luk'yanchuk and V. Ternovsky, "Light scattering by a thin wire with a surface-plasmon resonance: bifurcations of 
the Poynting vector field," Physical Review B, vol. 73, no. 23, Article ID 235432, 2006.

[4] B. S. Luk'yanchuk, M. I. Tribelsky, Z. B. Wang et al., "Extraordinary scattering diagram for nanoparticles near plasmon resonance frequencies," Applied Physics A, vol. 89, no. 2, pp. 259-264, 2007.

[5] M. I. Tribelsky, S. Flach, A. E. Miroshnichenko, A. V. Gorbach, and Y. S. Kivshar, "Light scattering by a finite obstacle and fano resonances," Physical Review Letters, vol. 100, no. 4, Article ID 043903, 2008.

[6] W. Fang, J. Y. Xu, A. Yamilov et al., "Large enhancement of spontaneous emission rates of InAs quantum dots in GaAs microdisks," Optics Letters, vol. 27, no. 11, pp. 948-950, 2002.

[7] C. Qiu, L. Gao, J. D. Joannopoulos, and M. Soljačić, "Light scattering from anisotropic particles: propagation, localization, and nonlinearity," Laser and Photonics Reviews, vol. 4, no. 2, pp. 268-282, 2010.

[8] S. Zhang, Y. Xiong, G. Bartal, X. Yin, and X. Zhang, "Magnetized plasma for reconfigurable subdiffraction imaging," Physical Review Letters, vol. 106, no. 24, 2011.

[9] J. Yao, X. Yang, X. Yin, G. Bartal, and X. Zhang, "Threedimensional nanometer-scale optical cavities of indefinite medium," Proceedings of the National Academy of Sciences of the United States of America, vol. 108, no. 28, pp. 11327-11331, 2011.

[10] S. Liu, W. Lu, Z. Lin, and S. T. Chui, "Molding reflection from metamaterials based on magnetic surface plasmons," Physical Review B, vol. 84, no. 4, 2011.

[11] S. Liu, J. Du, Z. Lin, R. X. Wu, and S. T. Chui, "Formation of robust and completely tunable resonant photonic band gaps," Physical Review B, vol. 78, no. 15, 2008.

[12] S. Liu, W. Chen, J. Du, Z. Lin, S. T. Chui, and C. T. Chan, "Manipulating negative-refractive behavior with a magnetic field," Physical Review Letters, vol. 101, no. 15, Article ID 157407, 2008.

[13] Y. Poo, R.-X. Wu, Z. Lin, Y. Yang, and C. T. Chan, "Experimental realization of self-guiding unidirectional electromagnetic edge states," Physical Review Letters, vol. 106, no. 9, 2011.

[14] J. Roth and M. J. Dignam, "Scattering and extinction cross sections for a spherical particle coated with an oriented molecular layer," The Journal of the Optical Society of America, vol. 63, no. 3, pp. 308-311, 1973.

[15] J. C. Monzon, "Three-dimensional field expansion in the most general rotationally symmetric anisotropic material: application to scattering by a sphere," IEEE Transactions on Antennas and Propagation, vol. 37, no. 6, pp. 728-735, 1989.

[16] A. D. Kiselev, V. Y. Reshetnyak, and T. J. Sluckin, "Light scattering by optically anisotropic scatterers: T-matrix theory for radial and uniform anisotropies," Physical Review E, vol. E65, no. 5, Article ID 056609, pp. 1-16, 2002.

[17] M. F. Yanik, H. Cinar, H. N. Cinar, A. D. Chisholm, Y. Jin, and A. Ben-Yakar, "Functional regeneration after laser axotomy," Nature, vol. 432, no. 7019, p. 822, 2004.

[18] R. D. Weglein, "A model for predicting acoustic material signatures," Applied Physics Letters, vol. 34, no. 3, pp. 179-181, 1979.

[19] J. Kushibiki, A. Ohkubo, and N. Chubachi, "Anisotropy detection in sapphire by acoustic microscope using line-focus beam," Electronics Letters, vol. 17, no. 15, pp. 534-536, 1981.

[20] A. C. Guo, J. R. Petrella, J. Kurtzberg, and J. M. Provenzale, "Evaluation of white matter anisotropy in Krabbe disease with diffusion tensor MR imaging: initial experience," Radiology, vol. 218, no. 3, pp. 809-815, 2001.
[21] J. B. Pendry, D. Schurig, and D. R. Smith, "Controlling electromagnetic fields," Science, vol. 312, no. 5781, pp. 17801782, 2006.

[22] X. Q. Sheng and Z. Peng, "Analysis of scattering by large objects with off-diagonally anisotropic material using finite element-boundary integral-multilevel fast multipole algorithm," IET Microwaves, Antennas and Propagation, vol. 4, pp. 492-500, 2010.

[23] W. Ren, "Contributions to the electromagnetic wave theory of bounded homogeneous anisotropic media," Physical Review E, vol. 47, no. 1, pp. 664-673, 1993.

[24] Y. Geng, X. Wu, and L.-W. Li, "Analysis of electromagnetic scattering by a plasma anisotropic sphere," Radio Science, vol. 38, no. 6, pp. 12/1-12/12, 2003.

[25] Y. L. Geng and X. B. Wu, "A Plane Electromagnetic Wave Scattering By a Ferrite Sphere," Journal of Electromagnetic Waves and Applications, vol. 18, no. 2, pp. 161-179, 2004.

[26] Y.-L. Geng, X.-B. Wu, L.-W. Li, and B.-R. Guan, "Mie scattering by a uniaxial anisotropic sphere," Physical Review E, vol. 70, no. 5, article 056609, pp. 1-8, 2004.

[27] Y. L. Geng and C. W. Qiu, "Analytical spectral-domain scattering theory of a general gyrotropic sphere," Materials Science. In press, http://arxiv.org/abs/1102.4057.

[28] Y.-L. Geng, "Scattering of a plane wave by an anisotropic ferrite-coated conducting sphere," IET Microwaves, Antennas and Propagation, vol. 2, no. 2, pp. 158-162, 2008.

[29] B. Stout, M. Nevière, and E. Popov, "Mie scattering by an anisotropic object. Part I. Homogeneous sphere," Journal of the Optical Society of America A, vol. 23, no. 5, pp. 1111-1123, 2006.

[30] B. Stout, M. Nevière, and E. Popov, "Mie scattering by an anisotropic object. Part II. Arbitrary-shaped object: differential theory," Journal of the Optical Society of America A, vol. 23, no. 5, pp. 1124-1134, 2006.

[31] J. Roth and M. J. Dignam, "Scattering and extinction cross sections for a spherical particle coated with an oriented molecular layer," Journal of the Optical Society of America A, vol. 63, no. 3, pp. 308-311, 1973.

[32] W. L. Ong, "New solutions to EM scattering by a gyrotropic sphere," Bachelor Thesis, National University of Singapore, 2010.

[33] Y. L. Geng, "Application of Spherical Vector Wave Function to Electromagnetic Scattering by Anisotropic Media," $P h D$. Thesis, Xi'an, China, Xidian University, 2006.

[34] C. T. Tai, Dyadic Green's Functions in Electromagnetic Theory, IEEE Press, New York, NY, USA, 1993.

[35] N. K. Uzunoglu, P. G. Cottis, and J. G. Fikioris, "Excitation of electromagnetic waves in a gyroelectric cylinder," IEEE Transactions on Antennas and Propagation, vol. 33, no. 1, pp. 90-99, 1985.

[36] S. N. Papadakis, N. K. Uzunoglu, and N. Christos, "Scattering of a plane wave by a general anisotropic dielectric ellipsoid," Journal of the Optical Society of America A, vol. 7, no. 6, pp. 991-997, 1990.

[37] W. C. Chew and Y. M. Wang, "Fast algorithm for solution of a scattering problem using a recursive aggregate T-matrix method," Microwave and Optical Technology Letters, vol. 3, no. 5, pp. 164-169, 1990.

[38] D. Sarkar and N. J. Halas, "General vector basis function solution of Maxwell's equations," Physical Review E, vol. 56, no. 1, pp. 1102-1112, 1997.

[39] D. Sarkar, Vector basis function solution of Maxwell's equations, Ph.D. thesis, Rice University, Houston, Tex, USA, 1996. 
[40] S. Liu, "Electromagnetic properties for three dimensional anisotropic photonic crystal," PhD. Thesis, Fudan University, Shanghai, China, 2007.

[41] Z. Lin and S. T. Chui, "Electromagnetic scattering by optically anisotropic magnetic particle," Physical Review E, vol. 69, no. 5, article 056614, pp. 1-14, 2004.

[42] S. Liu and Z. Lin, "Opening up complete photonic bandgaps in three-dimensional photonic crystals consisting of biaxial dielectric spheres," Physical Review E, vol. 73, no. 6, 2006.

[43] L. Gao, T. H. Fung, K. W. Yu, and C. W. Qiu, "Electromagnetic transparency by coated spheres with radial anisotropy," Physical Review E, vol. 78, no. 4, article 046609, 2008.

[44] C.-W. Qiu, A. Novitsky, H. Ma, and S. Qu, "Electromagnetic interaction of arbitrary radial-dependent anisotropic spheres and improved invisibility for nonlinear-transformation-based cloaks," Physical Review E, vol. 80, no. 1, 2009.

[45] C. W. Qiu, L. Hu, B. Zhang, B. I. Wu, S. G. Johnson, and J. D. Joannopoulos, "Spherical cloaking using nonlinear transformations for improved segmentation into concentric isotropic coatings," Optics Express, vol. 17, no. 16, pp. 1346713478, 2009.

[46] C.-W. Qiu, L. Hu, X. Xu, and Y. Feng, "Spherical cloaking with homogeneous isotropic multilayered structures," Physical Review E, vol. 79, no. 4, article 047602, 2009.

[47] A. Novitsky, C. W. Qiu, and S. Zouhdi, "Transformationbased spherical cloaks designed by an implicit transformationindependent method: theory and optimization," New Journal of Physics, vol. 11, Article ID 113001, 2009.

[48] B. S. Luk'yanchuk and C.-W. Qiu, "Enhanced scattering efficiencies in spherical particles with weakly dissipating anisotropic materials," Applied Physics A, vol. 92, no. 4, pp. 773-776, 2008.

[49] Y. X. Ni, D. L. Gao, Z. F. Sang, L. Gao, and C. W. Qiu, "Influence of spherical anisotropy on the optical properties of plasmon resonant metallic nanoparticles," Applied Physics A, vol. 102, no. 3, pp. 673-679, 2011.

[50] W.-J. Chen, J. C. W. Lee, J.-W. Dong, C.-W. Qiu, and H.-Z. Wang, "Fano resonance of three-dimensional spiral photonic crystals: paradoxical transmission and polarization gap," Applied Physics Letters, vol. 98, no. 8, article 081116, 2011.

[51] C.-W. Qiu, L.-W. Li, T.-S. Yeo, and S. Zouhdi, "Scattering by rotationally symmetric anisotropic spheres: potential formulation and parametric studies," Physical Review E, vol. 75, no. 2, 2007.

[52] C. F. Bohren and D. R. Huffman, Absorption and Scattering of Light by Small Particles, John Wiley \& Sons, New York, NY, USA, 1998.

[53] C.-W. Qiu, L.-W. Li, Q. Wu, and T.-S. Yeo, "Field representations in general gyrotropic media in spherical coordinates," IEEE Antennas and Wireless Propagation Letters, vol. 4, no. 1, pp. 467-470, 2005.

[54] A. Novitsky and L. Barkovsky, "Matrix approach for light scattering from a multilayered rotationally symmetric bianisotropic sphere," Physical Review A, vol. 77, no. 3, Article ID 033849, 2008.

[55] G. Arfken and H. J. Weber, Mathematical Method for Physicists, Elsevier Academic Press, 6th edition, 2005. 

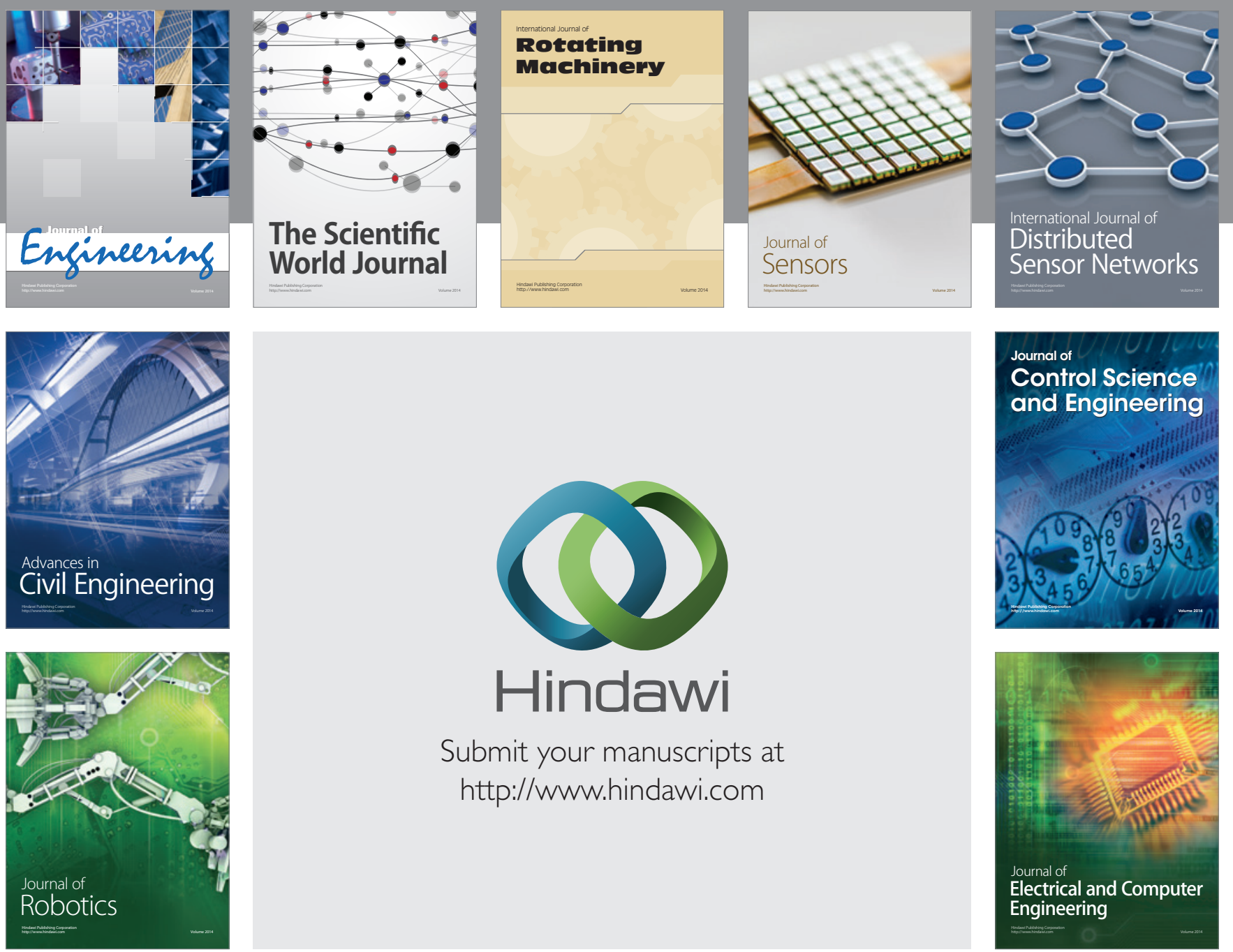

Submit your manuscripts at

http://www.hindawi.com
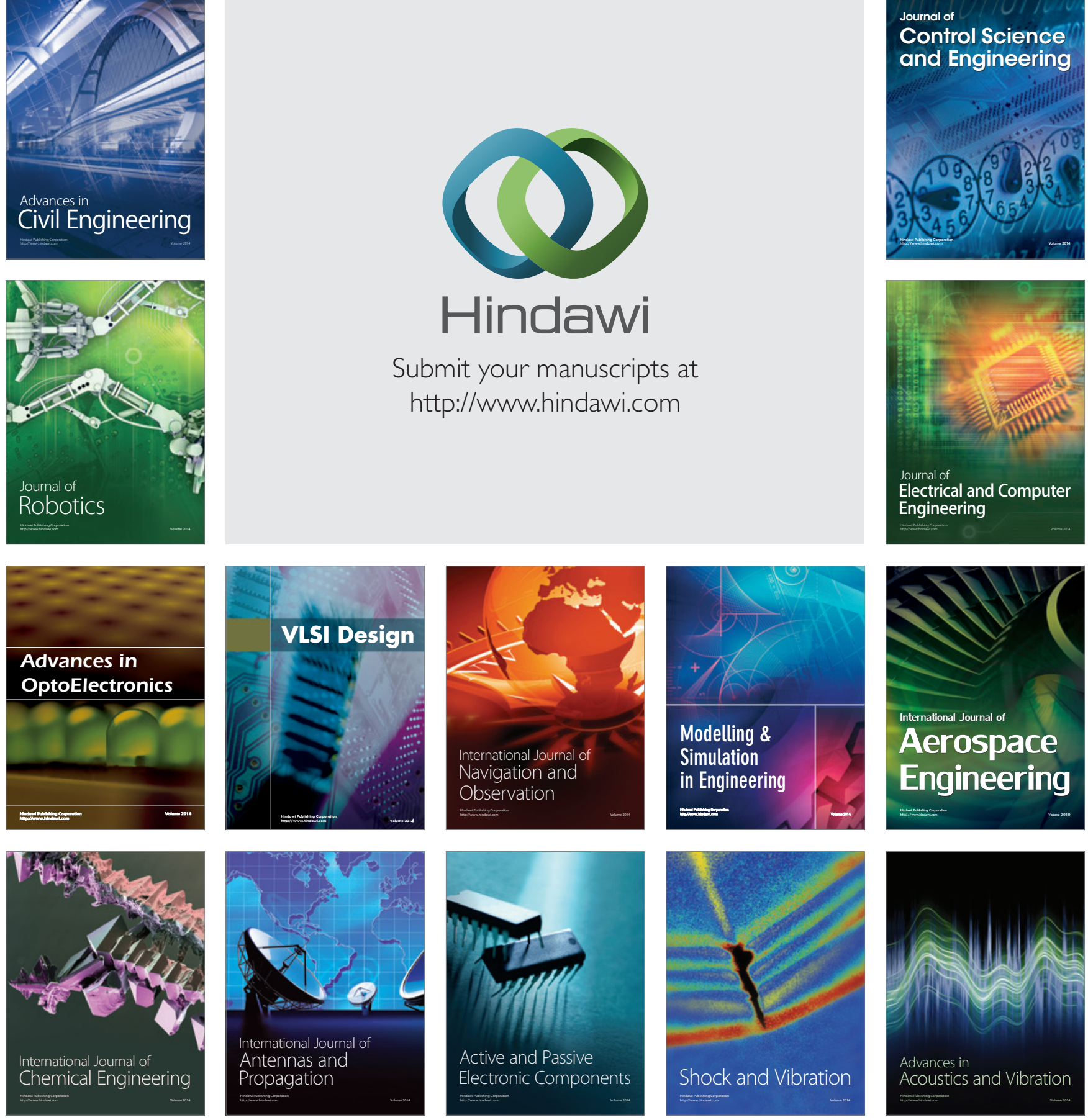